OUTP-01-20-P

\title{
The high energy cosmic ray spectrum from relic particle decay
}

\author{
Subir Sarkar* and Ramon Toldrà ${ }^{\dagger}$ \\ Theoretical Physics, University of Oxford, 1 Keble Road, Oxford OX1 3NP, UK \\ (submitted to Nucl. Phys. B)
}

\begin{abstract}
It has been speculated that the recently detected ultra-high energy cosmic rays may originate from the decays of relic particles with mass of order $10^{12}$ $\mathrm{GeV}$ clustered in the halo of our Galaxy. This hypothesis can be tested through forthcoming measurements of the spectra of both high energy cosmic nucleons and neutrinos, which are determined in this model by the physics of QCD fragmentation, with no astrophysical uncertainties. We evolve fragmentation spectra measured at LEP energies up to the scale of the decaying particle mass by numerical solution of the DGLAP equations. This enables incorporation of the effects of supersymmetry on the development of the cascade and we also allow for decays into many-particle states. The calculated spectral shape agrees well with present cosmic ray data beyond the GreisenZatsepin-Kuzmin energy.
\end{abstract}

98.70.Sa, 14.80.-j, 95.35.+d, 13.87.Fh

*sarkar@thphys.ox.ac.uk

†toldra@thphys.ox.ac.uk 


\section{INTRODUCTION}

Within the last decade, the Fly's Eye [1] and its successor HiRes [2], as well as the Akeno Giant Air Shower Array (AGASA) [3], have detected over 70 ultra-high energy cosmic rays (UHECRs) with energies measured reliably to be in excess of $E_{\mathrm{GZK}} \simeq 4 \times 10^{19} \mathrm{eV}$ - the Greisen-ZatsepinKuzmin (GZK) 'cutoff' energy 泡. The older air shower arrays at Volcano Ranch [5], Haverah Park [6], and Yakutsk [7] have added another 45 (recalibrated) post-GZK events. At energies above $E_{\mathrm{GZK}}$, the typical range of protons falls rapidly because of interactions with cosmic microwave background photons [8,9], becoming as low as $\sim 20 \mathrm{Mpc}$ at $3.2 \pm 0.9 \times 10^{20} \mathrm{eV}$, the highest energy event observed by Fly's Eye; the range is even smaller for heavy nuclei 10.1. For photons with energy above $E_{\mathrm{GZK}}$, the dominant opacity is due to scattering on the extragalactic radio background which is poorly known experimentally [16]; the mean free path is estimated to be $\sim 1-5 \mathrm{Mpc}$ at $10^{21} \mathrm{eV}$ [17. 2] There is no restrictive constraint on the range if the UHECR primaries are neutral and weakly interacting, viz. neutrinos which have only a tiny probability for scattering on the relic cosmic neutrino background [22,23]. 3] This also means however that neutrinos cannot initiate the observed airshowers as their cross-section for deep inelastic scattering on nucleons is far too small [27. Th Thus all the available evidence suggests that cosmic rays with energies $E>E_{\mathrm{GZK}}$ are protons, in which case they must originate within the Local Supercluster.

\footnotetext{
${ }^{1}$ The shower elongation rate measured by Fly's Eye [1].11] and HiRes [12] indicates a change in composition from heavier nuclei towards nucleons at the highest energies. Muon data from AGASA [13] are consistent with this trend when analysed using the same hadronic interaction model [14,15].

${ }^{2}$ The highest energy event [1] had a depth of maximum of $815_{-35}^{+45} \mathrm{~g} \mathrm{~cm}^{-2}$ cf. the expected value of $1075 \mathrm{~g} \mathrm{~cm}^{-2}$ for a photon primary [18]. The muon content of horizontal showers in the Haverah Park data implies an upper bound of $65 \%$ on the photon component, and a (hadronic interaction model dependent) upper bound of $\sim 50 \%$ on the iron nuclei content, of post-GZK UHECRs [19]. It should be emphasised however that a complete understanding of air shower development is still lacking, so the composition of UHECRs is not definitively established yet [20,21].

${ }^{3}$ However if the background neutrinos have mass of $\sim 0.1-1 \mathrm{eV}$, then UHE cosmic neutrinos can annihilate resonantly on these to generate 'Z-bursts' which create photons and nucleons above

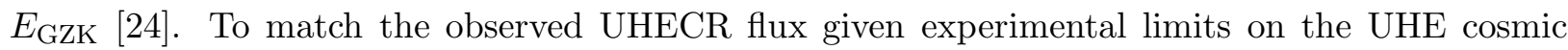
neutrino flux [25] requires however a substantial increase in the local density of relic neutrinos [26].

${ }^{4}$ Neutrinos may interact more strongly if dramatic new physics is invoked e.g. extra dimensions and quantum gravity at the $\mathrm{TeV}$ scale [28]. However explicit calculations indicate so far that the increased cross-section would still be inadequate to create the observed air-showers [29]. This possibility is testable through studies of penetrating airshowers [30] and upward-going muons [31].

${ }^{5} \mathrm{~A}$ suggested correlation between UHECR arrival directions and cosmologically distant radio sources [32] motivates the possibility that the primary is a new heavy hadron (e.g. a supersymmetric $u d s$-gluino state) with a higher GZK cutoff [33]. The correlation has however been questioned [34]; moreover there are stringent experimental bounds on new stable strongly interacting particles [35].
} 
However they cannot originate in the Galactic disk since significant anisotropies would then be expected at post-GZK energies [36], while the observed sky distribution is consistent with isotropy 37,38. 5 A number of doublet and triplet events, collimated within the experimental angular resolution of a few degrees, have been observed but the chance probability of these arising from an isotropic distribution is estimated to be of order $10 \%$ [40, hence statistically not significant.]

There have been a plethora of suggestions as to the possible origin of UHECRs, which may be broadly classified into 'top-down' or 'bottom-up' models. In the former class, the extreme energies are supposedly provided by the decay of relic topological defects (TD) 41,43,45 or super-massive particles 46 49. In the latter class, the UHECRs are assumed to have been accelerated up from low energies in astrophysical sites such as gamma-ray bursts [50], active galaxies [51] or super-massive black holes in dormant quasars [52]. In fact there are severe difficulties with accelerating particles to the highest observed energies by any known astrophysical mechanism [53]. Moreover even if such sources are distributed nearby like the observed galaxies, the injection spectrum must be harder than $\mathrm{d} N / \mathrm{d} E \propto E^{-2}$ in order to overcome the GZK energy losses and match the measured UHECR flux [9,54], so the energetic demands are considerable. Gamma-ray bursters may possibly have the required energy but, being more abundant at high redshift, cannot yield the locally observed UHECR flux [55]. There are a few active galaxies within the 'GZK sphere' but even if they can accelerate UHECR these would need to be deflected through rather large angles (and isotropised) by the intergalactic magnetic field [56]; this requires the field strength to be $\sim 10^{3}$ times stronger than the upper limit of order nanogauss usually inferred from observational bounds on Faraday rotation in distant radio sources [57]. There is ongoing discussion concerning the possibility of such high fields [58, as well as of specific sources such as Cen A 59 61 and Virgo A 62, 63. Although there is no compelling astrophysical model for post-GZK UHECRs, some of these suggestions are interesting in their own right and can be tested with forthcoming data.

In this paper, we investigate the spectral signature of 'top-down' models. Relic topological defects will in general be cosmologically distant and release particles at the GUT-scale, resulting in a typically excessive flux of secondary low energy $\gamma$-rays as the primaries interact with intergalactic radiation fields 44,45]. So we favour the possibility that UHECRs originate from the decay of supermassive relic particles which, being cold dark matter (CDM), are locally clustered in the halo of our Galaxy, thus naturally evading the GZK-cutoff and also presenting an approximately isotropic distribution 48,49 . Such particles must have a mass $\gtrsim 10^{12} \mathrm{GeV}$ to account for the highest energy event, and a lifetime exceeding the age of the universe. A physically well-motivated candidate with both the required mass and lifetime is the "crypton" - the analogue of a hadron in the hidden sector of supersymmetry breaking in string theories [64].8] It has been noted that such particles can readily be produced with a cosmologically interesting abundance through the time-varying gravitational field at the end of inflation [66], or possibly, during (re)heating after inflation [67].

\footnotetext{
${ }^{6}$ At around $10^{18}$ eV Fly's Eye [37] and AGASA 39] have detected anisotropy towards the Galactic plane, consistent with the usual belief that cosmic rays at these energies have a galactic origin.

${ }^{7}$ However if only the paired events within $\pm 10^{0}$ of the supergalactic plane are considered, the chance probability that they arise from an isotropic distribution is less than $1 \%$ 40.

${ }^{8}$ Other particle candidates which may be sufficiently long-lived have also been proposed 65].
} 
A key test of this model is the expected small anisotropy due to the offset of the Sun from the centre of the Galaxy [68].9] Some authors have argued [60,70] that present limits on the anisotropy already place severe constraints on the model; however a recent detailed analysis of the expected anisotropy signal [71] of post-GZK UHECRs shows it to be both compatible with all present data and moreover capable of being definitively detected by the Pierre Auger Project which is presently under construction [72], and by the proposed Extreme Universe Space Observatory [73]. These experiments will provide a very substantial increase in the presently meagre statistics of post-GZK UHECRs and also be able to distinguish between incident photon, neutrino and nucleon primaries. This is clearly an opportune time to make predictions of the expected spectrum and composition of UHECRs in the decaying dark matter (DDM) model.

\section{THE DECAYING DARK MATTER MODEL}

The essential idea 448,49] here is that because of gravitational clustering, very massive relic particles would naturally have a density in the halo of our Galaxy which is enhanced by a factor of $\sim 10^{4}$ over the cosmic average. This is approximately the same factor by which the distance to the horizon exceeds the halo radius, so if these particles decay to produce UHECRs the flux from cosmologically distant regions will be at best comparable to the flux from our halo even if there is no absorption [74. Given the GZK attenuation, the extragalactic flux of protons and photons in this model would be quite negligible compared to the flux from the Galactic halo, whereas for neutrinos there would be an extragalactic component comparable to the galactic one [23].

The injection spectrum produced by the decay of a population of superheavy dark matter particles $X$, with number density $n_{X}$, is proportional to the inclusive decay width of $X$ :

$$
\Phi^{\text {halo }}(E)=\frac{n_{X}}{\tau_{X}} \frac{1}{\Gamma_{X}} \frac{\mathrm{d} \Gamma(X \rightarrow h+\ldots)}{\mathrm{d} E},
$$

where we have taken the lifetime of $X$ to be longer than the age of the universe, $\tau_{X} \gg t_{0} \sim 10^{10}$ yr. Photons produced in the galactic halo can interact with the galactic low frequency radio background on their way to the Earth; the number of high energy photons reaching the Earth may then be significantly reduced. However the radio background is presently very poorly known [16] so we do not include photon processing in the halo in this work. We consider a spherical halo of radius $R_{\text {halo }}$ and uniform density $n_{X}^{\text {halo }}$ (see Ref. [71] for a thorough study of UHECR and halo models). The galactic halo contribution to the UHECR spectrum is then given by

$$
J^{\text {halo }}(E)=\frac{1}{4 \pi} R_{\text {halo }} \Phi^{\text {halo }}(E) .
$$

In order to calculate the inclusive decay width, some assumptions have to be made about the microphysics of $X$ decay. It is usually assumed in top-down models (i.e. for both TD and DDM) that the initial decay is into a parton-antiparton pair. Any angular dependence of the decay amplitude is immaterial since the flux at Earth is close to isotropic. Therefore in the following Section ([II]) we shall consider decays into quarks and gluons (and also their superpartners when allowing for the presence of supersymmetry).

\footnotetext{
${ }^{9}$ If the clustering in the arrival directions turns out to be statistically significant, it may be an indication of clumpiness in the halo dark matter distribution, as is indeed expected for CDM [69].
} 


\section{EVOLUTION OF FRAGMENTATION FUNCTIONS}

\section{A. Inclusive Decay Width}

For a particle $X$ with mass $M_{X}$, decaying into partons which hadronise into particles of type $h$ (carrying a fraction $x$ of the maximum available momentum $M_{X} / 2$, and a fraction $z$ of the parton momentum), the inclusive decay width can be factorised as [76]

$$
\frac{1}{\Gamma_{X}} \frac{\mathrm{d} \Gamma(X \rightarrow h+\ldots)}{\mathrm{d} x}=\left.\sum_{a} \int_{x}^{1} \frac{\mathrm{d} z}{z} \frac{1}{\Gamma_{a}} \frac{\mathrm{d} \Gamma_{a}\left(y, \mu^{2}, M_{X}^{2}\right)}{\mathrm{d} y}\right|_{y=x / z} D_{a}^{h}\left(z, \mu^{2}\right) .
$$

The first factor, the decay width of $X$ into parton $a, \mathrm{~d} \Gamma_{a} / \mathrm{d} y$, is calculable in perturbation theory; in lowest order and for 2-body decay it is proportional to $\delta(1-y)$. The second factor, the nonperturbative $D_{a}^{h}$, is the fragmentation function (FF) for particles of type $h$ from partons of type $a$. Both factors depend on the factorisation scale $\mu$. The dependence on $\mu$ of the physical inclusive decay width would cancel out if the calculation could be carried out to all orders in perturbation theory but in a finite-order calculation some dependence on $\mu$ remains to orders higher than those calculated. The choice $\mu \sim M_{X}$ is the most appropriate [77]. Every perturbative term in the above factorisation depends also on an arbitrary renormalization scale; following the usual convention, we take the renormalization scale to equal the factorisation scale.

The cancellation of the dependence on $\mu$ between the perturbative factors and the FFs constrains the latter to satisfy the Dokshitzer-Gribov-Lipatov-Altarelli-Parisi (DGLAP) equations [78, 79]. Given experimental data on hadronisation at some low energy scale, say $M_{Z}$, an initial set of fragmentation functions $D_{a}^{h}\left(x, M_{Z}^{2}\right)$ can be extracted. These can then be evolved using the DGLAP equations to obtain the FFs at some higher scale $D_{a}^{h}\left(x, M_{X}^{2}\right)$. This evolution gives rise to scaling violations in the $x$-dependence of the inclusive decay width of $X$ in exactly the same way that scale-dependence of structure functions and FFs produce scaling violations in experimentally measured hadronic cross-sections [76].

The DGLAP equations can be written as

$$
\frac{\partial D_{a}^{h}\left(x, \mu^{2}\right)}{\partial \ln \mu^{2}}=\sum_{b} \frac{\alpha_{s}\left(\mu^{2}\right)}{2 \pi} P_{b a}\left(x, \alpha_{s}\left(\mu^{2}\right)\right) \otimes D_{b}^{h}\left(x, \mu^{2}\right),
$$

where $\alpha_{s}\left(\mu^{2}\right)$ is the strong coupling constant and $P_{b a}\left(x, \alpha_{s}\right)$ is the splitting function for the parton branching $a \rightarrow b$. Here the convolution of two functions $A(x)$ and $B(x)$ is defined as

$$
A(x) \otimes B(x) \equiv \int_{x}^{1} \frac{\mathrm{d} z}{z} A(z) B\left(\frac{x}{z}\right) .
$$

The splitting functions can be expanded perturbatively:

$$
P_{b a}\left(x, \alpha_{s}\right)=P_{b a}(x)+\mathcal{O}\left(\alpha_{s}\right) .
$$

We limit our study to leading order in $\alpha_{s}$ and therefore ignore $\mathcal{O}\left(\alpha_{s}\right)$ corrections to the splitting functions. It is also convenient to define the following dimensionless evolution parameter

$$
\tau \equiv \frac{1}{2 \pi b} \ln \frac{\alpha_{s}\left(\mu_{0}^{2}\right)}{\alpha_{s}\left(\mu^{2}\right)},
$$

$b$ being the coefficient in the leading order $\beta$-function governing the running of the strong coupling: $\beta\left(\alpha_{s}\right)=-b \alpha_{s}^{2}$. We take $D_{a}^{h}$ to represent the sum of particle $h$ and, if different, its antiparticle $\bar{h}$. 


\section{B. Standard Model Equations}

The Standard Model DGLAP equations for the evolution of fragmentation functions are wellknown [76,77]. There are two parton species: quarks $q_{k}, k=1, \ldots n_{\mathrm{F}}$, and gluons $g$, with $n_{\mathrm{F}}$ the total number of flavours. Conventionally one defines the following linear combinations (for ease of notation the superscript $h$ is omitted):

$$
\begin{aligned}
D_{q_{k}^{+}} & \equiv D_{q_{k}}+D_{\bar{q}_{k}}, \\
D_{q} & \equiv \sum_{k} D_{q_{k}^{+}}, \\
D_{q_{k}^{-}} & \equiv D_{q_{k}}-D_{\bar{q}_{k}}, \\
D_{Q_{k}} & \equiv D_{q_{k}^{+}}-\frac{1}{n_{\mathrm{F}}} D_{q} .
\end{aligned}
$$

The non-singlet functions $D_{q_{k}^{-}}$and $D_{Q_{k}}$ obey the equations

$$
\begin{gathered}
\partial_{\tau} D_{q_{k}^{-}}=P_{q q} \otimes D_{q_{k}^{-}}, \\
\partial_{\tau} D_{Q_{k}}=P_{q q} \otimes D_{Q_{k}},
\end{gathered}
$$

while the evolution of the singlet function $D_{q}$ is coupled to that of the gluon function $D_{g}$ as

$$
\partial_{\tau}\left(\begin{array}{c}
D_{q} \\
D_{g}
\end{array}\right)=\left(\begin{array}{cc}
P_{q q} & 2 n_{\mathrm{F}} P_{g q} \\
P_{q g} & P_{g g}
\end{array}\right) \otimes\left(\begin{array}{c}
D_{q} \\
D_{g}
\end{array}\right) .
$$

The splitting functions were calculated in Refs. [78, [76]. Given the FFs at some initial scale $\mu_{0}$ for

the quarks $q_{k}$ and gluon $g$, we can now determine their evolved values at some other scale $\mu$ to leading order in $\alpha_{s}$, using Eqs. (12 14).

\section{Supersymmetric Equations}

In a supersymmetric (SUSY) model, besides the quarks and gluons one has their superpartners: squarks $s_{k}$ and gluinos $\lambda$. In addition to the linear combinations (811) we now define

$$
\begin{aligned}
D_{s_{k}^{+}} & \equiv D_{s_{k}}+D_{\bar{s}_{k}}, \\
D_{s} & \equiv \sum_{k} D_{s_{k}^{+}}, \\
D_{s_{k}^{-}} & \equiv D_{s_{k}}-D_{\bar{s}_{k}}, \\
D_{S_{k}} & \equiv D_{s_{k}^{+}}-\frac{1}{n_{\mathrm{F}}} D_{s} .
\end{aligned}
$$

The non-singlet function $D_{q_{k}^{-}}$and $D_{s_{k}^{-}}$evolve together, as do $D_{Q_{k}}$ and $D_{S_{k}}$ :

$$
\begin{aligned}
\partial_{\tau}\left(\begin{array}{c}
D_{q_{k}^{-}} \\
D_{s_{k}^{-}}
\end{array}\right)=\left(\begin{array}{ll}
P_{q q} & P_{s q} \\
P_{q s} & P_{s s}
\end{array}\right) \otimes\left(\begin{array}{c}
D_{q_{k}^{-}} \\
D_{s_{k}^{-}}
\end{array}\right), \\
\partial_{\tau}\left(\begin{array}{c}
D_{Q_{k}} \\
D_{S_{k}}
\end{array}\right)=\left(\begin{array}{ll}
P_{q q} & P_{s q} \\
P_{q s} & P_{s s}
\end{array}\right) \otimes\left(\begin{array}{c}
D_{Q_{k}} \\
D_{S_{k}}
\end{array}\right) .
\end{aligned}
$$


The singlet functions for quarks and squarks, $D_{q}$ and $D_{s}$, are coupled to the gluon and gluino functions, $D_{g}$ and $D_{\lambda}$, as

$$
\partial_{\tau}\left(\begin{array}{c}
D_{q} \\
D_{g} \\
D_{s} \\
D_{\lambda}
\end{array}\right)=\left(\begin{array}{cccc}
P_{q q} & 2 n_{\mathrm{F}} P_{g q} & P_{s q} & 2 n_{\mathrm{F}} P_{\lambda q} \\
P_{q g} & P_{g g} & P_{s g} & P_{\lambda g} \\
P_{q s} & 2 n_{\mathrm{F}} P_{g s} & P_{s s} & 2 n_{\mathrm{F}} P_{\lambda s} \\
P_{q \lambda} & P_{g \lambda} & P_{s \lambda} & P_{\lambda \lambda}
\end{array}\right) \otimes\left(\begin{array}{c}
D_{q} \\
D_{g} \\
D_{s} \\
D_{\lambda}
\end{array}\right) .
$$

In leading order, Eqs. (19 21) allow us to calculate the fragmentation functions for all quark and squark flavours, gluons and gluinos at some scale $\mu$, given their values at some initial scale $\mu_{0}$.

Incidentally, using the following relationships among the SUSY splitting functions [80],

$$
\begin{aligned}
& P_{q q}+P_{s q}=P_{s s}+P_{q s}, \\
& P_{g q}+P_{\lambda q}=P_{\lambda s}+P_{g s}, \\
& P_{q g}+P_{s g}=P_{s \lambda}+P_{q \lambda}, \\
& P_{g g}+P_{\lambda g}=P_{\lambda \lambda}+P_{g \lambda},
\end{aligned}
$$

one can reduce the $4 \times 4$ matrix equation (21) to the $2 \times 2$ matrix equation

$$
\partial_{\tau}\left(\begin{array}{c}
D_{q}-D_{s} \\
D_{g}-D_{\lambda}
\end{array}\right)=\left(\begin{array}{cc}
P_{q q}-P_{q s} & 2 n_{\mathrm{F}}\left(P_{g q}-P_{g s}\right) \\
P_{q g}-P_{q \lambda} & P_{g g}-P_{g \lambda}
\end{array}\right) \otimes\left(\begin{array}{c}
D_{q}-D_{s} \\
D_{g}-D_{\lambda}
\end{array}\right) .
$$

The SUSY DGLAP equations have been given in the literature to leading order for structure functions [80,81]. Here we have presented their form for FFs. It is easy to see that (except for Eq. (26) ) one just needs to transpose the matrix elements, keeping the $n_{\mathrm{F}}$ factors in the same place, to move from structure function to fragmentation function equations. For Eq. (26), the relative minus sign between $q, s$ and $g, \lambda$ changes to a plus sign and there is a rearrangement of the splitting functions in the $2 \times 2$ matrix. The SUSY splitting functions were calculated in Refs. 80,81.

\section{Numerical Evolution: Algorithm and Initial Conditions}

Several numerical algorithms and their implementation codes are available to solve the set of integro-differential DGLAP equations for structure functions when quarks and gluons are the only particles inside the hadrons 82,83]. The supersymmetric evolution of structure functions has been considered in Ref. 84]. These codes are suitable for collider physics but are rather unwieldy to use for studying cosmic ray production. We have generalised the Laguerre method [82] to include SUSY evolution for fragmentation functions and written a numerical code to calculate cosmic ray production from superheavy particle decay. The main idea of the Laguerre method is to expand Eq. (四) in Laguerre polynomials; the coefficients of the series expansion are given by simple recursive relations appropriate for computer calculations. These recursive relations have been given so far for the 1-dimensional case (Eqs. 12 13) and the 2-dimensional case (Eq. 14). However to study the general SUSY evolution requires one to work out the recursive relations for the 4-dimensional case (Eq. 21). This is not a trivial step if one follows the procedure given for lower dimensional cases in the literature. Hence we have calculated the general recursive relations for any dimension. The details of this procedure, as well as our numerical code, will be given elsewhere 85.

We begin with FFs at the energy scale $\mu_{0}=M_{Z}$ and evolve them to the final energy scale $\mu=M_{X}$ using Eq. (四). For the initial fragmentation function of baryons, $D_{a}^{p}\left(x, M_{Z}^{2}\right)+D_{a}^{n}\left(x, M_{Z}^{2}\right)$, 
we adopt the fit performed in Ref. [86] to LEP hadronic data [88], as shown in Fig. 1. For photons and neutrinos we generate initial data at the $Z$ peak using the QCD Monte Carlo event generator HERWIG 89. Comparison with LEP data shows that although HERWIG overproduces baryons at high $x$ 86, 87, its photon and meson output at the $Z$ peak matches the experimental spectra remarkably well. Since neutrinos mainly come from charged pion and kaon decays, one can thus be confident in taking the HERWIG generated FF for neutrinos as the initial condition for the evolution. There is also a sizable contribution from heavy flavour decays to the neutrino spectrum at high $x$ which is explicitly taken into account by HERWIG. Figure 2 shows our initial spectra at the energy scale $M_{Z}$ for photons and neutrinos together with LEP data for photons.

We assume flavour universality in the decay of $X$, hence we consider only the coupled singlet quark and gluon evolution equations (14) for the Standard Model (SM), and the coupled singlet quark, gluon, singlet squark and gluino evolution equations (21) for a SUSY model. A (s)parton is not included in the evolution as long as the energy scale is lower than its mass; when the threshold for its production is crossed, it is added to the evolution equations with an initially vanishing $\mathrm{FF}$ and it is assumed to be a relativistic particle.

In the SM case we evolve the $q$ and $g$ initial fragmentation functions from $M_{Z}$ to $M_{t}$, the top quark mass, with the number of flavours set to $n_{\mathrm{F}}=5$, and then evolve from $M_{t}$ to $M_{X}$ with $n_{\mathrm{F}}=6$. (However assuming $n_{\mathrm{F}}=6$ in the whole range from $M_{Z}$ to $M_{X}$ does not introduce any significant difference in the final spectrum.)

In the SUSY case we evolve the $q$ and $g$ initial fragmentation functions from $M_{Z}$ to the supersymmetry breaking scale $M_{\mathrm{SUSY}}>M_{t}$ using the SM equations to obtain $D_{i}^{h}\left(x, M_{\mathrm{SUSY}}^{2}\right)$, with $i=q, g$. Then we take $D_{i}^{h}\left(x, M_{\mathrm{SUSY}}^{2}\right), i=q, g$, and $D_{j}^{h}\left(x, M_{\mathrm{SUSY}}^{2}\right)=0, j=s, \lambda$, and evolve them from $M_{\mathrm{SUSY}}$ to $M_{X}$ using the SUSY equations. All spartons are taken to be degenerate with a common mass $M_{\text {SUSY }}$. (In the context of structure functions, a SUSY model with different masses for $s$ and $\lambda$ was studied [84], finding no significant difference with models having the same mass for all superpartners. One might expect the same result to hold for FFs.)

For very small $x$ values, $x \lesssim 0.001$, coherent gluon emission becomes important. In this regime Eq. (㺼) no longer holds and the kernel of the integral must be modified as $D_{a}\left(x / z, \mu^{2}\right) \rightarrow$ $D_{a}\left(x / z, z^{2} \mu^{2}\right)$. This is the Modified Leading Logarithm Approximation (MLLA) 76, 90] which has been used by other authors to calculate the cosmic ray spectrum in top-down models 43, 48, 91. However the MLLA spectrum cannot be normalised since a very small (and uncertain) fraction of the total energy is released in this kinematic region; moreover in the context of the DDM model for a particle mass of order the hidden-sector scale, the observed highest energy cosmic rays correspond to large values of $x$ 49,92. There are also other corrections to the DGLAP equations arising from a more rigorous treatment of threshold effects [77] and, of course, next-to-leading order corrections to $\alpha_{s}$ which we have not considered. Nevertheless, the level of precision afforded by the DGLAP equations in leading order, when calculating scaling violations in the inclusive decay of $X$, is quite adequate for comparison with the present experimental data on UHECRs.

For $x \sim 1$ there are large uncertainties (see Fig. 1) in the experimental determination of the FFs [88. Generating values for these using HERWIG requires rather large amounts of computational time since very few particles fall in the bins with $x>0.8$, if the number of simulated events is kept to a reasonable number [49]. Furthermore, the algorithms which solve the DGLAP equations show poor convergence in this regime. These problems motivate an alternative approach to calculating high $x$ fragmentation. One can take a power-law fit to the experimental data at $M_{Z}$, and then use the Mellin transform technique [76] to calculate its evolved value at $M_{X}$. If at $\tau_{0}$ one has the fit 
$D_{a}\left(x, \tau_{0}\right)=K(1-x)^{k}$, where $K$ and $k$ are constants, then when $x \rightarrow 1$ we find

$$
D_{a}(x, \tau)=K\left[\frac{\alpha_{s}\left(\tau_{0}\right)}{\alpha_{s}(\tau)}\right]^{B_{a}} \frac{\Gamma(k+1)}{\Gamma\left(A_{a}\left(\tau-\tau_{0}\right)+k+1\right)}(1-x)^{k+A_{a}\left(\tau-\tau_{0}\right)},
$$

where in the SM

$$
\begin{aligned}
& A_{q}=2 C_{\mathrm{F}}, \quad B_{q}=\frac{C_{\mathrm{F}}}{2 \pi b}\left(\frac{3}{2}-2 \gamma\right), \\
& A_{g}=2 C_{\mathrm{A}}, \quad B_{g}=\frac{1}{2 \pi b}\left[C_{\mathrm{A}}\left(\frac{11}{6}-2 \gamma\right)-\frac{2}{3} T_{\mathrm{R}} n_{\mathrm{F}}\right],
\end{aligned}
$$

and in a SUSY model

$$
\begin{aligned}
& A_{q}=A_{s}=2 C_{\mathrm{F}}, \quad B_{q}=B_{s}=\frac{C_{\mathrm{F}}}{2 \pi b}(1-2 \gamma) \\
& A_{g}=A_{\lambda}=2 C_{\mathrm{A}}, \quad B_{g}=B_{\lambda}=\frac{1}{2 \pi b}\left[C_{\mathrm{A}}\left(\frac{3}{2}-2 \gamma\right)-T_{\mathrm{R}} n_{\mathrm{F}}\right] .
\end{aligned}
$$

The $S U(3)$ fundamental and adjoint group factors are respectively $C_{\mathrm{F}}=4 / 3, C_{\mathrm{A}}=3, b$ is the leading order coefficient of the $\beta$-function (7), $T_{\mathrm{R}}=1 / 2$, and $\gamma=0.57721 \ldots$ is the Euler constant. Eq. (27) shows that for $x \rightarrow 1$, a power-law $D_{a}$ evolves at higher energy into a power-law with a bigger power index: the higher the energy the faster the drop in $(1-x)$.

\section{E. Numerical Evolution: Results and Discussion}

First we present the evolution of the baryon fragmentation function in the SM. In Fig. 3 we show the fragmentation functions for baryons $(p$ and $n)$ from quarks and gluons at the scale $M_{Z}$

as measured at LEP, and their evolved shape at $M_{X}=10^{10}, 10^{12}$ and $10^{14} \mathrm{GeV}$. (Following the standard convention we always plot the quantity $x^{3} D_{a}\left(x, \mu^{2}\right)$, which is proportional to the cosmic ray energy spectrum $E^{3} J(E)$.) As the final scale increases, the number of particles grows at low $x$ and decreases at high $x$ as is well-known from many previous studies of scaling violations.

Next we compare the SUSY evolution of FFs with SM evolution. In Fig. 1 we show the common initial baryon FF at $M_{Z}$, its shape after SM evolution up to $M_{X}=10^{12} \mathrm{GeV}$, and its evolved shape at the same final scale after SUSY has been switched on at $M_{\text {SUSY }}=400 \mathrm{GeV}$. It is clear that the SUSY curve has evolved further than the SM curve, chiefly due to the different running of $\alpha_{s}\left(\mu^{2}\right)$. In a SUSY model $\alpha_{s}$ decreases with increasing energy more slowly than in the SM because of the increased contribution to the $\beta$-function from the SUSY partners. Since the rate of change $\partial_{\ln \mu^{2}} D_{a}\left(x, \mu^{2}\right)$ is proportional to $\alpha_{s}$ (see Eq. 团), a bigger $\alpha_{s}$ translates into a larger amount of evolution. In other words, given the same initial and final scales we obtain $\tau_{\mathrm{SUSY}}\left(M_{X}\right)>\tau_{\mathrm{SM}}\left(M_{X}\right)$, using Eq. (7).

Figure 5 shows the quark and gluon FFs at $M_{Z}$, their evolved values at $M_{X}=10^{12} \mathrm{GeV}$ using the SUSY equations for scales larger than $M_{\mathrm{SUSY}}$, and the radiatively generated squark and gluino functions, all at the same final scale $M_{X}$. We find that starting from vanishing values at $M_{\mathrm{SUSY}}$ the squark and gluino functions start to grow and catch up rapidly with the quark and gluon functions, respectively, at small $x$. This behaviour can be understood qualitatively if one bears in mind that at low $x$ the leading splitting function for quarks is $2 n_{\mathrm{F}} P_{g q} \sim 4 n_{\mathrm{F}} C_{\mathrm{F}} / x$, which is equal to the leading splitting function for squarks $2 n_{\mathrm{F}} P_{g s} \sim 4 n_{\mathrm{F}} C_{\mathrm{F}} / x$. For gluons and gluinos the leading splitting functions tend as well to a common value, $P_{g g} \sim 2 C_{\mathrm{A}} / x$ and $P_{g \lambda} \sim 2 C_{\mathrm{A}} / x$, which is however different from that of quarks and squarks. 
SUSY evolution does not depend strongly on the chosen supersymmetry breaking scale $M_{\mathrm{SUSY}}$. In Fig. 5 we show the curves obtained taking $M_{\text {SUSY }}=200,400 \mathrm{GeV}$ and $1 \mathrm{TeV}$. The higher the value of $M_{\mathrm{SUSY}}$, the less evolved the final curves for $q$ and $g$. This follows from our earlier comparison of SM vs. SUSY evolution. If SUSY switches on later (higher $M_{\text {SUSY }}$ ) the energy range over which the SM equations hold is larger. As we have seen already, DGLAP evolution is slower when just the SM equations are employed.

The evolution of the photon and neutrino spectra can also be studied using the DGLAP equations. We take HERWIG simulated data for the initial FFs for photons and neutrinos (sum over all three flavours) at $M_{Z}$ and use the SM and SUSY evolution equations (14) and (21) to obtain their spectra at $M_{X}$. We plot in Fig. 6 the fragmentation functions for baryons, photons and neutrinos for a decaying particle of mass $M_{X}=10^{12} \mathrm{GeV}$, in both the SM and in a SUSY model with $M_{\mathrm{SUSY}}=400 \mathrm{GeV}$. As pointed out earlier we assume flavour symmetry in the decay of $X$; moreover all the calculated FFs are colour-weighted. Hence for the SM the total contribution to the FF for particle of type $h$ is just the sum of quark singlet and gluon FFs

$$
D^{h} \equiv D_{q}^{h}+D_{g}^{h}
$$

while in a SUSY model we take the sum of quark and squark singlets, gluon and gluino FFs

$$
D^{h} \equiv D_{q}^{h}+D_{g}^{h}+D_{s}^{h}+D_{\lambda}^{h}
$$

\section{ULTRA HIGH ENERGY COSMIC RAY SPECTRUM}

We can now translate the calculated fragmentation functions into the expected cosmic ray spectrum in order to confront the observational data. In the previous Section (III) we have calculated quark singlet and gluon functions for the SM, and quark singlet, gluon, squark singlet and gluino functions for SUSY. In the absence of a specific model for the different branching ratios we have weighted all the (s)parton contributions evenly but for colour weights as in Eqs. (32 33). Thus the quark contribution is the most important as seen from Figs. 3 and 5; in the SUSY case the squark contribution is also relevant for $x \leq 0.01$. For 2-body decay, $x=2 E / M_{X}$, so from Eqs. (11-3) we have the following expression for the flux of particle $h$ :

$$
E^{3} J^{\text {halo }}(E)=B x^{3} D^{h}\left(x, M_{X}^{2}\right) .
$$

We have multiplied the flux by $E^{3}$, as is usual, to emphasise the structure in the spectrum near the GZK energy. The normalisation factor $B$ is common for the galactic halo flux of baryons, neutrinos and photons, and determines the quantity $n_{X} / \tau_{X}$ (see Eq. 3).

Let us now compare the calculated cosmic ray flux to the published data from Fly's Eye [1], AGASA [3], Haverah Park [6] and Yakutsk [7]. In Ref. [75] these data have been carefully assessed for mutual consistency and appropriate adjustments made to the energy calibration, e.g. the original AGASA data [3] was reduced in energy by $10 \%$ to match the Akeno $1-\mathrm{km}^{2}$ array data which covers the better explored energy region $\sim 10^{15}-10^{18} \mathrm{eV}$ [93]. The authors recommend adoption of the following standard differential energy spectrum below the GZK energy, in the range $4 \times 10^{17} \mathrm{eV}<E<6.3 \times 10^{18} \mathrm{eV}$ :

$$
J(E)=(9.23 \pm 0.65) \times 10^{-33} \mathrm{~m}^{-2} \mathrm{~s}^{-1} \mathrm{sr}^{-1} \mathrm{eV}^{-1}\left(\frac{E}{6.3 \times 10^{18} \mathrm{eV}}\right)^{-3.20 \pm 0.05}
$$


with the spectrum flattening at higher energies as $J(E) \propto E^{-2.75 \pm 0.2}$ upto the GZK energy, and extending further to at least $3 \times 10^{20} \mathrm{eV}$ [75]. Thus the UHECR spectrum can naturally be interpreted [1] as the superposition of the 'low energy' component (35), and the new 'flat' component that extends into the post-GZK region. The former is presumably galactic in origin (consistent with the detection of anisotropy at $\sim 10^{18} \mathrm{eV}[37,39]$ ), while the latter is interpreted [49] as produced by the decay of a superheavy particle population in the galactic halo. Taking baryons to be the dominant primary UHECRs as indicated by experiment, the total flux is

$$
E^{3} J(E)=\frac{k}{E^{m}}+B x^{3} D^{\text {baryon }}\left(x, M_{X}^{2}\right),
$$

where the values of $k$ and $m$ can be read off Eq. (35). Note that since $D^{\text {baryon }}$ and $D^{\gamma}$ have a similar shape, taking photons to be the primaries would just alter the normalisation $B$.

We have performed a least-squares fit to the data. Figure 0 shows the best SM fit which corresponds to a mass $M_{X}=10^{12} \mathrm{GeV}$, with $\chi^{2}=132$ for 81 degrees of freedom. The low energy component (36) and the total spectrum are also shown. In calculating $\chi^{2}$ we ignore the highest energy point at $\sim 3.2 \times 10^{20} \mathrm{eV}$ which is based on a single event, hence has a large flux uncertainty. Nevertheless this event is important since it requires us to reject values of $M_{X}$ much below $10^{12} \mathrm{GeV}$ which cannot generate such an event, although they allow a reasonable fit to the rest of data. In Fig. 8 we plot the best SUSY fit taking a common mass of $M_{\mathrm{SUSY}}=400 \mathrm{GeV}$ for all spartons; the favoured decaying particle mass is now $M_{X}=5 \times 10^{12} \mathrm{GeV}$, with a slightly lower $\chi^{2}$ of 130 .

The assumption of 2-body decay may be rather naive for a superheavy particle like a crypton which is expected to decay through very high-order non-renormalisable operators 64. Many-body decay distributes the total energy $M_{X}$ among several particles and thus flattens the spectrum. We assume that many-body effects are purely kinematical and hence can be encapsulated in the phase space of the decay. In Appendix A we calculate $\rho_{n}(z)$, the probability density that one parton carries off a fraction $z$ of the total available energy per parton $M_{X} / 2$. For $n \geq 3$ we get

$$
\rho_{n}(z) \propto z(1-z)^{n-3} .
$$

To leading order in $\alpha_{s}$ the particle flux is then given by

$$
E^{3} J^{\text {halo }}(E)=B x^{3} \int_{x}^{1} \frac{\mathrm{d} z}{z} \rho_{n}\left(\frac{x}{z}\right) D^{h}\left(z, M_{X}^{2}\right) .
$$

In particular if $D^{h}\left(x, M_{X}^{2}\right) \propto(1-x)^{a\left(M_{X}^{2}\right)}$ as $x \rightarrow 1$, the differential particle flux decreases as $J^{\text {halo }}(x) \propto(1-x)^{a\left(M_{X}^{2}\right)+n-2}$.

For many-body $X$ decays the total flux is

$$
E^{3} J(E)=\frac{k}{E^{m}}+B x^{3} \int_{x}^{1} \frac{\mathrm{d} z}{z} \rho_{n}\left(\frac{x}{z}\right) D^{\text {baryon }}\left(z, M_{X}^{2}\right),
$$

where $n$ is the number of partons into which $X$ decays. In Fig. 9 we plot SUSY spectra with $M_{X}=10^{13} \mathrm{GeV}, M_{\mathrm{SUSY}}=400 \mathrm{GeV}$ and $n=2,8,16$. As $n$ increases the spectrum flattens since the average momentum is pushed to lower values of $x$ (see Appendix A). For $n=2,8,16$ we get $\chi^{2}=133,127,126$ respectively. Clearly many-body decays fit the data better which is encouraging as this would be natural in the crypton model [64. With the forthcoming improvement in event statistics a joint fit to the decaying particle mass and decay multiplicity would be warranted. 


\section{DISCUSSION}

We now compare these results to our previous work in which the fragmentation spectra were found by directly running HERWIG at the decaying particle mass scale [49, as well as to other work where numerical solutions to the DGLAP evolution were also reported [86, 95], and, finally, to a recently proposed independent method for calculating the fragmentation spectrum [96].

First it should be emphasised that most studies of 'top-down' models have employed the MLLA approximation to the fragmentation functions [43,45, 48,91] which, as noted earlier, is valid only at $x \ll 1$ where a very small (and uncertain) amount of energy is released, and is thus incapable of being normalised as was done in these papers. Other studies [42] have used a convenient (and properly normalised) parameterisation of the fragmentation function based on PETRA data at $\sim 20 \mathrm{GeV}$ 41] which, however, does not account for the large scaling violations in evolving up to the very high energies of interest here. Moreover in both these approaches it was assumed that the ratio of pions to baryons in the cascade remains fixed at its low energy value of 20:1 at all energies. (In reality one would expect the FF of say neutrinos to evolve differently from that of baryons due to new processes at high energies e.g. decays of heavy flavours.) All these studies focussed on a decaying particle mass of $\sim 10^{13-16} \mathrm{GeV}$, close to the GUT scale.

These difficulties were emphasised in our previous work [49] where HERWIG was used to make the first realistic estimate of the fragmentation function for super-massive particle decay, including the expected large scaling violations. It was shown that the UHECR data favoured a decaying particle mass of $\mathcal{O}\left(10^{12}\right) \mathrm{GeV}$ - close to the 'hidden sector' scale of SUSY breaking rather than to the GUT scale. However this work suffered itself from the fact that HERWIG overproduces baryons by a factor of $\sim 3$ at high $x$ (see Fig. 1); moreover it did not account for the effects of supersymmetry on the development of the cascade. Both these issues were addressed in Ref. 86] which suggested that the way forward was to evolve measured fragmentation functions at LEP up to the decaying particle mass. For the SUSY case, sparton FFs were generated at $10^{4} \mathrm{GeV}$ using the event generator PYTHIA [94] and the evolution done for a supergravity model with parameters $M_{0}=800 \mathrm{GeV}, M_{1 / 2}=200 \mathrm{GeV}, A_{0}=0, \tan \beta=10, \operatorname{sgn}(\mu)=+$. Apart from highlighting the high- $x$ baryon overproduction problem in the previous work 49 for the SM case, this work showed that the effects of supersymmetry could be important in that the most likely decaying particle mass increased from $\sim 10^{12} \mathrm{GeV}$ for the SM to $\sim 10^{13} \mathrm{GeV}$ for SUSY 86]. Subsequently the DGLAP equations were also solved in Ref. 95] following a similar procedure but these authors favoured the possibility that the decaying particles are uniformly distributed in intergalactic space rather than being clustered in the halo. Then the decay spectrum undergoes GZK processing, developing a 'bump' below the putative cutoff; by fitting this to the (unnormalised) data from AGASA, Fly's Eye and Haverah Park they found an even higher decaying particle mass of $\sim 10^{15} \mathrm{GeV}[95$. (For the physically better motivated case of a clustered halo population, their best-fit mass is $10^{12} \mathrm{GeV}$ in agreement with previous work 49, 86, , but since the FFs for this mass were not presented, we cannot compare their results with ours in detail here.) Finally in Ref. 96] a new Monte Carlo simulation for jet fragmentation was presented and used to calculate the SUSY FFs for a $10^{12} \mathrm{GeV}$ mass particle; however the SM FFs were not presented."ण0

\footnotetext{
${ }^{10}$ The authors acknowledge errors in their previous analytic calculation of the SUSY FF in the MLLA approximation [91, which was used in several studies of TD models [43,45].
} 
In Fig. 10 we compare the (flavour-averaged) FFs of baryons, photons and neutrinos for a $10^{12} \mathrm{GeV}$ mass decaying particle obtained from HERWIG [49], with the results from (SM) DGLAP evolution in Ref. [86], as well as in the present study. The baryon overproduction problem at high $x$ is evident in the HERWIG results; in fact photons and neutrinos now dominate at all energies. Secondly the results obtained by DGLAP evolution agree reasonably well, keeping in mind that Ref. [86] incorporated gluon coherence [76] and also next-to-leading order corrections for both $\alpha_{s}$ evolution and the splitting functions, which we have not done in the present work.

In Fig. 11 we compare the (flavour-averaged) FFs for baryons, photons and neutrinos obtained from (SUSY) DGLAP evolution in Ref. 86] and in present work, with the results from the new fragmentation code of Ref. 96]. There are significant differences between the results. Note that we have taken sparton FFs to be zero at threshold rather than generating them with PYTHIA above threshold as was done in Ref. [86]; this is presumably the major reason for the difference in our results and reflects the present degree of theoretical uncertainty in this approach. The results obtained by the new Monte Carlo simulation for jet fragmentation 966 differ significantly from both DGLAP evolution calculations. Since the SM FFs were not presented in this work we cannot check whether the new code 96] can reproduce the (consistent) results for SM DGLAP evolution discussed above. In view of these discrepancies, further work is clearly necessary to calculate the spectrum reliably in the supersymmetric case.

In summary we have developed a new numerical code to solve the DGLAP evolution equations for fragmentation functions in heavy particle decay. We have used this to calculate the expected spectra of baryons, photons and neutrinos from the decays of hypothetical metastable dark matter particles of mass $10^{12} \mathrm{GeV}$ clustered in the halo of our Galaxy, both for the case of Standard Model evolution and for a simple supersymmetric model. The shape of the fragmentation spectrum (of either baryons or photons) fits rather well the new component of ultra-high energy cosmic rays extending beyond the GZK energy; the fit improves if the effects of supersymmetry and, more importantly, of many-body decays are taken into account.

It is sometimes stated that the decaying particle model is already in conflict with observations because the dominant particles in the fragmentation cascades are predicted to be photons rather than baryons which are favoured by experiment [19]. However as mentioned earlier the photons may be significantly attenuated in their passage to Earth due to interactions on the radio background in the halo, particularly if there is a fossil radio 'ghost' around our Galaxy [97. Whether this is indeed possible, subject to the EGRET bound on the low energy $\gamma$-rays which will be created by such electromagnetic cascades, will be discussed by us elsewhere. Also of interest is the expected flux of high energy neutrinos which can be detected in forthcoming experiments such as ANTARES [98] and ICECUBE [99]. Most importantly the Pierre Auger Observatory [72] will significantly increase the statistics of UHECRs enabling a better measurement of the spectrum, as well as of the composition and (an)isotropy. It will soon be possible to definitively test the exciting possibility that cosmic rays have already shown us a glimpse of physics far beyond the Standard Model.

\section{ACKNOWLEDGMENTS}

We are grateful to Peter Richardson and Mike Seymour for assistance in the use of HERWIG and John Ellis, Neil Rubin, Alan Watson and Bryan Webber for many discussions. We would also

like to thank Zoltan Fodor, Michael Kachelrieß, Sandor Katz and Motohiko Nagano for helpful correspondence. R.T. is supported by a Marie Curie Fellowship No. HPMF-CT-1999-00268. 


\section{APPENDIX A: MANY-BODY DECAY KINEMATICS}

We briefly review how to calculate the phase space $R_{n}$ for the decay of $X$ into $n$ partons (see Ref. [100] for further details); this is needed to calculate the probability density of obtaining a decay parton with energy $z M_{X} / 2$.

From a kinematical point of view the decay $X \rightarrow a_{1}+a_{2}+a_{3}+\ldots+a_{n}$ can be decomposed recursively as a 2-body decay $X \rightarrow a_{1}+A_{1}$ where $A_{1}$ is a particle with 4-momentum equal to the sum of 4-momenta of particles $a_{2}, a_{3} \ldots a_{n}$. Then $A_{1}$ is decayed into two particles $A_{1} \rightarrow a_{2}+A_{2}$ where $A_{2}$ has as 4-momentum the sum of the 4-momenta of $a_{3} \ldots a_{n}$, and so on up to the decay $A_{n-2} \rightarrow a_{n-1}+a_{n}$. This recursive procedure allows the phase space integral $R_{n}\left(M_{X}, m_{1}, \ldots m_{n}\right)$, $n \geq 3$, to be written as

$$
\begin{aligned}
R_{n}= & \int_{\left(m_{2}+m_{3}+\ldots+m_{n}\right)^{2}}^{\left(M_{X}-m_{1}\right)^{2}} \frac{\mathrm{d} M_{1}^{2}}{M_{1}^{2}} R_{2}\left(M_{X}, m_{1}, M_{1}\right) \int_{\left(m_{3}+\ldots+m_{n}\right)^{2}}^{\left(M_{1}-m_{2}\right)^{2}} \frac{\mathrm{d} M_{2}^{2}}{M_{2}^{2}} R_{2}\left(M_{1}, m_{2}, M_{2}\right) \\
& \ldots \int_{\left(m_{n-1}+m_{n}\right)^{2}}^{\left(M_{n-3}-m_{n-2}\right)^{2}} \frac{\mathrm{d} M_{n-2}^{2}}{M_{n-2}^{2}} R_{2}\left(M_{n-3}, m_{n-2}, M_{n-2}\right) \times R_{2}\left(M_{n-2}, m_{n-1}, m_{n}\right),
\end{aligned}
$$

where $M_{i}$ is the invariant mass of $A_{i}$ and $R_{2}\left(m_{a}, m_{b}, m_{c}\right)$ is the phase space integral for the 2-body decay $a \rightarrow b+c$ :

$$
\begin{aligned}
R_{2} & =\frac{1}{8 m_{a}} \lambda^{1 / 2}\left(m_{a}^{2}, m_{b}^{2}, m_{c}^{2}\right) \int \mathrm{d} \Omega, \\
\lambda(x, y, z) & \equiv x^{2}+y^{2}+z^{2}-2 x y-2 y z-2 x z .
\end{aligned}
$$

We shall study massless partons for which Eq. (A1) reduces to

$$
\begin{gathered}
R_{n} \propto \int_{0}^{M_{X}^{2}} \frac{\mathrm{d} M_{1}^{2}}{M_{1}^{2}}\left(M_{X}^{2}-M_{1}^{2}\right) \int_{0}^{M_{1}^{2}} \frac{\mathrm{d} M_{2}^{2}}{M_{2}^{2}}\left(M_{1}^{2}-M_{2}^{2}\right) \\
\ldots \int_{0}^{M_{n-3}^{2}} \frac{\mathrm{d} M_{n-2}^{2}}{M_{n-2}^{2}}\left(M_{n-3}^{2}-M_{n-2}^{2}\right) M_{n-2}^{2} .
\end{gathered}
$$

Let $\rho_{n}(z)$ be the probability density to get parton $a_{i}$ with an energy fraction $z=2 E_{i} / M_{X}\left(M_{X} / 2\right.$ is the maximum energy that a parton can carry, whatever $n$ is). By symmetry this probability is independent of the parton considered. Let us choose parton $a_{1}$. Then $z=1-M_{1}^{2} / M_{X}^{2}$ and

$$
\begin{aligned}
\rho_{n}(z) d z \propto & \frac{\mathrm{d} M_{1}^{2}}{M_{1}^{2}}\left(M_{X}^{2}-M_{1}^{2}\right) \int_{0}^{M_{1}^{2}} \frac{\mathrm{d} M_{2}^{2}}{M_{2}^{2}}\left(M_{1}^{2}-M_{2}^{2}\right) \\
& \ldots \int_{0}^{M_{n-3}^{2}} \frac{\mathrm{d} M_{n-2}^{2}}{M_{n-2}^{2}}\left(M_{n-3}^{2}-M_{n-2}^{2}\right) M_{n-2}^{2} .
\end{aligned}
$$

The multiple integration can easily be done to obtain

$$
\rho_{n}(z) \mathrm{d} z \propto\left(M_{X}^{2}-M_{1}^{2}\right)\left(M_{1}^{2}\right)^{n-3} \mathrm{~d} M_{1}^{2} \propto z(1-z)^{n-3} \mathrm{~d} z .
$$

After normalisation we finally obtain:

$$
\begin{aligned}
& \rho_{2}(z)=\delta(1-z), \\
& \rho_{n}(z)=(n-1)(n-2) z(1-z)^{n-3}, \quad n \geq 3 .
\end{aligned}
$$

We plot this distribution in Fig. 12. As the number of final particles $n$ increases, the total energy $M_{X}$ has to be shared between more particles and consequently less energy goes to each one of them. The probability density peaks at smaller values of $z$ as $n$ grows, accordingly the mean value of $z$ decreases as $\langle z\rangle_{n}=2 / n$. 


\section{REFERENCES}

[1] D.J. Bird et al., Phys. Rev. Lett. 71 (1993) 3401, Astrophys. J. 424 (1994) 491, 441 (1995) 144.

[2] T. Abu-Zayyad et al., Proc. ICRC 99, Salt Lake City, eds. B.L. Dingus et al.(AIP, 2000) Vol.3, p.264.

[3] M. Takeda et al., Phys. Rev. Lett. 81 (1998) 1163;

N. Hayashida et al., Astrophys. J. 522 (1999) 225.

[4] K. Greisen, Phys. Rev. Lett. 16 (1966) 748;

G.T. Zatsepin, V.A. Kuzmin, Sov. Phys. JETP Lett. 4 (1966) 78;

[5] J. Linsley, Phys. Rev. Lett. 10 (1963) 146.

[6] M.A. Lawrence, R.J.O. Reid, A.A. Watson, J. Phys. G17 (1991) 773.

[7] B.N. Afanasiev et al., Proc. Intern. Symp. on Extremely High Energy Cosmic Rays, Tokyo, ed. M. Nagano (Univ of Tokyo, 1996) p.32;

M.I. Pravdin et al., Proc. ICRC 99, Salt Lake City, eds. B.L. Dingus et al.(AIP, 2000) Vol.3, p.292.

[8] T. Stanev, R. Engel, A. Mücke, R.J. Protheroe, J.P. Rachen, Phys. Rev. D62 (2000) 093005.

[9] F.A. Aharonian, J.W. Cronin, Phys. Rev. D50 (1996) 1892.

[10] L.N. Epele, E. Roulet, JHEP 9810 (1998) 009;

F.W. Stecker, M.H. Salamon, Astrophys. J. 512 (1999) 521.

[11] L.K. Ding et al., Astrophys. J. 474 (1997) 490.

[12] T. Abu-zayaad et al., Phys. Rev. Lett. 84 (2000) 4276.

[13] N. Hayashida et al., J. Phys. G21 (1995) 1101.

[14] B.R. Dawson, R. Meyhandan, K.M. Simpson, Astropart. Phys. 9 (1998) 331.

[15] M. Nagano, D. Heck, K. Shinozaki, N. Inoue, J. Knapp, Astropart. Phys. 13 (2000) 277.

[16] A.H. Bridle, Mon. Not. R. Astron. Soc. 136 (1967) 14;

T.A. Clark, L.W. Brown, J.K. Alexander, Nature 228 (1970) 847.

[17] R.J. Protheroe, P.A. Johnson, Astropart. Phys. 4 (1996) 253 (Erratum-ibid.5 (1996) 215);

R.J. Protheroe, P.L. Biermann, Astropart. Phys. 6 (1996) 45 (Erratum-ibid.7 (1997) 181).

[18] F. Halzen, R.A. Vázquez, T. Stanev, H.P. Vankov, Astropart. Phys. 3 (1995) 151.

[19] M. Ave, J.A. Hinton, R.A. Vázquez, A.A. Watson, E. Zas, Phys. Rev. Lett. 85 (2000) 2244.

[20] T.K. Gaisser, Nucl. Phys. (Proc. Suppl.) 52B (1997) 10;

J. Knapp, Nucl. Phys. (Proc. Suppl.) 75A (1999) 89;

C. Pajares, D. Sousa, R.A. Vázquez, Phys. Rev. Lett. 86 (2001) 1674.

[21] L.A. Anchordoqui, M. Kirasirova, T.P. McCauley, S. Reucroft, J.D. Swain, Phys. Lett. B492 (2000) 237.

[22] T. Weiler, Phys. Rev. Lett. 49 (1982) 234.

[23] V.S. Berezinsky, Nucl. Phys. B380 (1992) 478;

P. Gondolo, G. Gelmini, S. Sarkar, Nucl. Phys. B392 (1993) 111.

[24] T. Weiler, Astropart. Phys. 11 (1999) 303;

D. Fargion, B. Mele, A. Salis, Astrophys. J. 517 (1999) 725.

[25] J.J. Blanco-Pillado, R.A. Vázquez, E. Zas, Phys. Rev. D61 (2000) 123003.

[26] S. Yoshida, G. Sigl, S. Lee, Phys. Rev. Lett. 81 (1998) 5505.

[27] R. Gandhi, C. Quigg, M.H. Reno, I. Sarcevic, Phys. Rev. D58 (1998) 093009.

[28] S. Nussinov, R. Shrock, Phys. Rev. D59 (1999) 105002; 
G. Domokos, S. Kovesi-Domokos, Phys. Rev. Lett. 82 (1999) 1366;

P. Jain, D.W. McKay, S. Panda, J.P. Ralston, Phys. Lett. B484 (2000) 267.

[29] M. Kachelrieß, M. Plümacher, Phys. Rev. D62 (2000) 103006;

F. Cornet, J.I. Illana, M. Masip, Phys. Rev. Lett. 86 (2001) 4235.

[30] C. Tyler, A.V. Olinto, G. Sigl, Phys. Rev. D63 (2001) 055001;

L. Anchordoqui et al., Phys. Rev. D63 (2001) 124009.

[31] J. Alvarez-Muñiz, F. Halzen, T. Han, D. Hooper, hep-ph/0107057.

[32] G.R. Farrar, P.L. Biermann, Phys. Rev. Lett. 81 (1998) 3579, 83 (1999) 2472;

but see, C.M. Hoffman, Phys. Rev. Lett. 83 (1999) 2471.

[33] D.J.H. Chung, G.R. Farrar, E.W. Kolb, Phys. Rev. D57 (1998) 4606;

I.F.M. Albuquerque, G.R. Farrar, E.W. Kolb, Phys. Rev. D59 (1999) 015021.

[34] G. Sigl, D. Torres, L. Anchordoqui, G. Romero, Phys. Rev. D63 (2001) 081302.

[35] D.E. Groom et al., Eur. Phys. J. C15 (2000) 1.

[36] A.A. Lee, R.W. Clay, J. Phys. G21 (1995) 1743;

T. Stanev, Astrophys. J. 479 (1997) 290;

G.A. Medina Tanco, E.M. de Gouveia Dal Pino, J.E. Horvath, Astrophys. J. 492 (1998) 200.

[37] D.J. Bird et al., Astrophys. J. 511 (1999) 739.

[38] M. Takeda et al., Astrophys. J. 522 (1999) 225.

[39] N. Hayashida et al., Astropart. Phys. 10 (1999) 303.

[40] Y. Uchihori et al., Astropart. Phys. 13 (2000) 151;

N. Hayashida et al., Phys. Rev. Lett. 77 (1996) 1000.

[41] C.T. Hill, Nucl. Phys. B224 (1983) 469.

[42] C.T. Hill, D.N. Schramm, T.P. Walker, Phys. Rev. D36 (1987) 1007;

P. Bhattacharjee, C.T. Hill, D.N. Schramm, Phys. Rev. Lett. 69 (1992) 567;

P. Bhattacharjee, G. Sigl, Phys. Rev. D51 (1995) 4079;

V. Berezinsky, A. Vilenkin, Phys. Rev. Lett. 79 (1997) 5202.

[43] V. Berezinsky, P. Blasi, A. Vilenkin, Phys. Rev. D58 (1998) 103515.

[44] R.J. Protheroe, T. Stanev, Phys. Rev. Lett. 77 (1996) 3708 (Erratum-ibid.78 (1997) 3420).

[45] G. Sigl, S. Lee, P. Bhattacharjee, S. Yoshida, Phys. Rev. D59 (1999) 043504.

[46] P.H. Frampton, S.L Glashow, Phys. Rev. Lett. 44 (1980) 1481;

J. Ellis, G. Steigman, T.K. Gaisser, Nucl. Phys. B177 (1981) 427.

[47] V.A. Kuzmin, V.A. Rubakov, Phys. Atom. Nucl. 61 (1998) 1028.

[48] V. Berezinsky, M. Kachelrieß, A. Vilenkin, Phys. Rev. Lett. 79 (1997) 4302.

[49] M. Birkel, S. Sarkar, Astropart. Phys. 9 (1998) 297.

[50] E. Waxman, Phys. Rev. Lett. 75 (1995) 386;

M. Vietri, Astrophys. J. 453 (1995) 883.

[51] J.P. Rachen and P.L. Biermann, Astron. Astrophys. 272 (1993) 161;

C.A. Norman, D.B. Melrose and A. Achterberg, Astrophys. J. 454 (1995) 60.

[52] E. Boldt, P. Ghosh, Mon. Not. R. Astron. Soc. 307 (1999) 491.

[53] R.D. Blandford, Phys. Scr. T85 (2000) 191;

A.M. Hillas, Ann. Rev. Astron. Astrophys. 22 (1984) 425.

[54] G. Medina Tanco, Astrophys. J. 510 (1999) L91;

M. Blanton, P. Blasi, A.V. Olinto, Astropart. Phys. 15 (2001) 275.

[55] F.W. Stecker, Astropart. Phys. 14 (2000) 207.

[56] J.W. Elbert, P. Sommers, Astrophys. J. 441 (1995) 151. 
[57] P.P. Kronberg, Rept. Prog. Phys. 57 (1994) 325;

J.P. Vallee, Fund. Cosm. Phys. 19 (1997) 1.

[58] D. Ryu, H. Kang, P.L. Biermann, Astron. Astrophys. 335 (1998) 19;

G. Sigl, M. Lemoine, P. Biermann, Astropart.Phys. 10 (1999) 141;

P. Blasi, S. Burles, A.V. Olinto, Astrophys. J. 514 (1999) L79;

G.R. Farrar, T. Piran, Phys. Rev. Lett. 84 (2000) 3527.

[59] L.A. Anchordoqui, H. Goldberg, T.J. Weiler, Phys. Rev. Lett. 87 (2001) 081101.

[60] G.R. Farrar, T. Piran, astro-ph/0010370.

[61] C. Isola, M. Lemoine, G. Sigl, astro-ph/0104289.

[62] E-J. Ahn, G. Medina-Tanco, P.L. Biermann, T. Stanev, astro-ph/9911123.

[63] P. Billoir, A. Letessier-Selvon, astro-ph/0001427;

D. Harari, S. Mollerach, E. Roulet, JHEP 0010 (2000) 047.

[64] J. Ellis, J.L. Lopez, D.V. Nanopoulos, Phys. Lett. B247 (1990) 257;

J. Ellis, G.B. Gelmini, J.L. Lopez, D.V. Nanopoulos, S. Sarkar, Nucl. Phys. B373 (1992) 399;

K. Benakli, J. Ellis, D.V. Nanopoulos, Phys. Rev. D59 (1999) 047301.

[65] S. Chang, C. Corianò and A.E. Faraggi, Nucl. Phys. B477 (1996) 65;

P.H. Frampton, B. Keszthelyi, Y.J. Ng, Int. J .Mod. Phys. D8 (1999) 117;

K. Hamaguchi, Y. Nomura, T. Yanagida, Phys. Rev. D58 (1998) 103503, Phys. Rev. D59 (1999) 063507;

C.P. Burgess, L.E. Ibáñez, F. Quevedo, Phys. Lett. B447 (1999) 257;

K. Hamaguchi, K.-I. Izawa, Y. Nomura, T. Yanagida, Phys. Rev. D60 (1999) 125009;

G. Dvali, Phys. Lett. B459 (1999) 489;

K. Hagiwara, Y. Uehara, Phys. Lett. B517 (2001) 383;

Y. Uehara, hep-ph/0107297.

[66] D. Chung, E.W. Kolb, A. Riotto, Phys. Rev. D59 (1999) 023501;

V. Kuzmin, I. Tkachev, Phys. Rev. D59 (1999) 123006;

D. Chung, P. Crotty, E.W. Kolb, A. Riotto, Phys. Rev. D64 (2001) 043503.

[67] D. Chung, E.W. Kolb, A. Riotto, Phys. Rev. D60 (1999) 063504.

[68] S.L. Dubovsky, P.G. Tinyakov, Sov. Phys. JETP. Lett. 68 (1998) 107;

G.A. Medina Tanco, A.A. Watson, Astropart. Phys. 12 (1999) 25;

V.S. Berezinsky, A.A. Mikhailov, Phys. Lett. 449 (1999) 237.

[69] P. Blasi, R.K. Sheth, Phys. Lett. B486 (2000) 233.

[70] A. Benson, A. Smialkowski, A. Wolfendale, Astropart. Phys. 10 (1999) 313;

T. Wibig, A.W. Wolfendale, J. Phys. G26 (2000) 1033.

[71] N.W. Evans, F. Ferrer, S. Sarkar, Astropart. Phys. (in press), astro-ph/0103085.

[72] The Pierre Auger Project (http://www.auger.org/)

[73] The Extreme Universe Space Observatory (http://www.ifcai.pa.cnr.it/ EUSD)

[74] A.M. Hillas, Nucl. Phys. (Proc. Suppl.) 75A (1999) 109.

[75] M. Nagano, A.A. Watson, Rev. Mod. Phys. 72 (2000) 689.

[76] R.K. Ellis, W.J. Stirling, B.R. Webber, QCD and Collider Physics (Cambridge University Press, 1996).

[77] P. Nason, B.R. Webber, Nucl. Phys. B421 (1994) 473 (Erratum-ibid.B480 (1996) 755).

[78] G. Altarelli, G. Parisi, Nucl. Phys. B126 (1977) 298.

[79] L.N. Lipatov, Sov. J. Nucl. Phys. 20 (1975) 94;

V.N. Gribov, L.N. Lipatov, Sov. J. Nucl. Phys. 15 (1972) 438; 
Yu.L. Dokshitzer, Sov. Phys. JETP 46 (1977) 641.

[80] C. Kounnas, D.A. Ross, Nucl. Phys. B214 (1983) 317.

[81] S.K. Jones, C.H. Llewellyn-Smith, Nucl. Phys. B217 (1983) 145.

[82] W. Furmański, R. Petronzio, Nucl. Phys. B195 (1982) 237.

[83] G.P. Ramsey, J. Comput. Phys. 60 (1985) 97;

S. Kumano, J.T. Londergan, Comput. Phys. Commun. 69 (1992) 373;

M.A.J. Botje, QCDNUM16: A Fast QCD Evolution Program, ZEUS note 97-066 (1997);

C. Corianò, C. Savkli, Comput. Phys. Commun. 118 (1999) 236.

[84] C. Corianò, hep-ph/0009227, hep-ph/0102164.

[85] R. Toldrà, hep-ph/0108127.

[86] N. Rubin, M. Phil. Thesis, Cavendish Laboratory, University of Cambridge (1999) (http://www.stanford.edu/ nrubin/Thesis.ps).

[87] A. Kupčo, Workshop on Monte Carlo Generators for HERA Physics, Hamburg, ed. T.A. Doyle et al.(DESY, 1999) p.292.

[88] R. Akers et al. (OPAL collab.) Z. Phys. C63 (1994) 181;

P. Abreu et al. (DELPHI collab.) Nucl. Phys. B444 (1995) 3;

R. Barate et al. (ALEPH collab.) Phys. Rep. 294 (1988) 1.

[89] G. Marchesini, B.R. Webber, G. Abbiendi, I.G. Knowles, M.H. Seymour, L. Stanco, Comput. Phys. Commun. 67 (1992) 465.

[90] Yu.L. Dokshitzer, V.A. Khoze, A.H. Mueller, S.I. Troyan, Basics of Perturbative QCD (Editions Frontieres, 1991);

V.A. Khoze, W. Ochs, Int. J. Mod. Phys. A12 (1997) 2949.

[91] V. Berezinsky, M. Kachelrieß, Phys. Lett. B434 (1998) 61.

[92] S. Sarkar, in COSMO-99: Proc. Third Intern. Workshop on Particle Physics and the Early Universe, eds. U Cotti et al.(World Scientific, 2000) p.77.

[93] N. Hayashida et al., Proc. Intern. Symp. on Extremely High Energy Cosmic Rays, Tokyo, ed. M. Nagano (Univ of Tokyo, 1996) p.17.

[94] T. Sjöstrand, Comp. Phys. Commun. 82 (1994) 74.

[95] Z. Fodor, S.D. Katz, Phys. Rev. Lett. 86 (2001) 3224.

[96] V. Berezinsky, M. Kachelrieß, Phys. Rev. D63 (2001) 034007.

[97] T.A. Enßlin, R.A. Sunyaev, astro-ph/0107432.

[98] ANTARES Collaboration, astro-ph/9907432.

[99] J. Alvarez-Muñiz, F. Halzen, Phys. Rev. D63 (2001) 037302.

[100] E. Byckling, K. Kajantie, Particle Kinematics, Wiley (1973). 


\section{FIGURES}

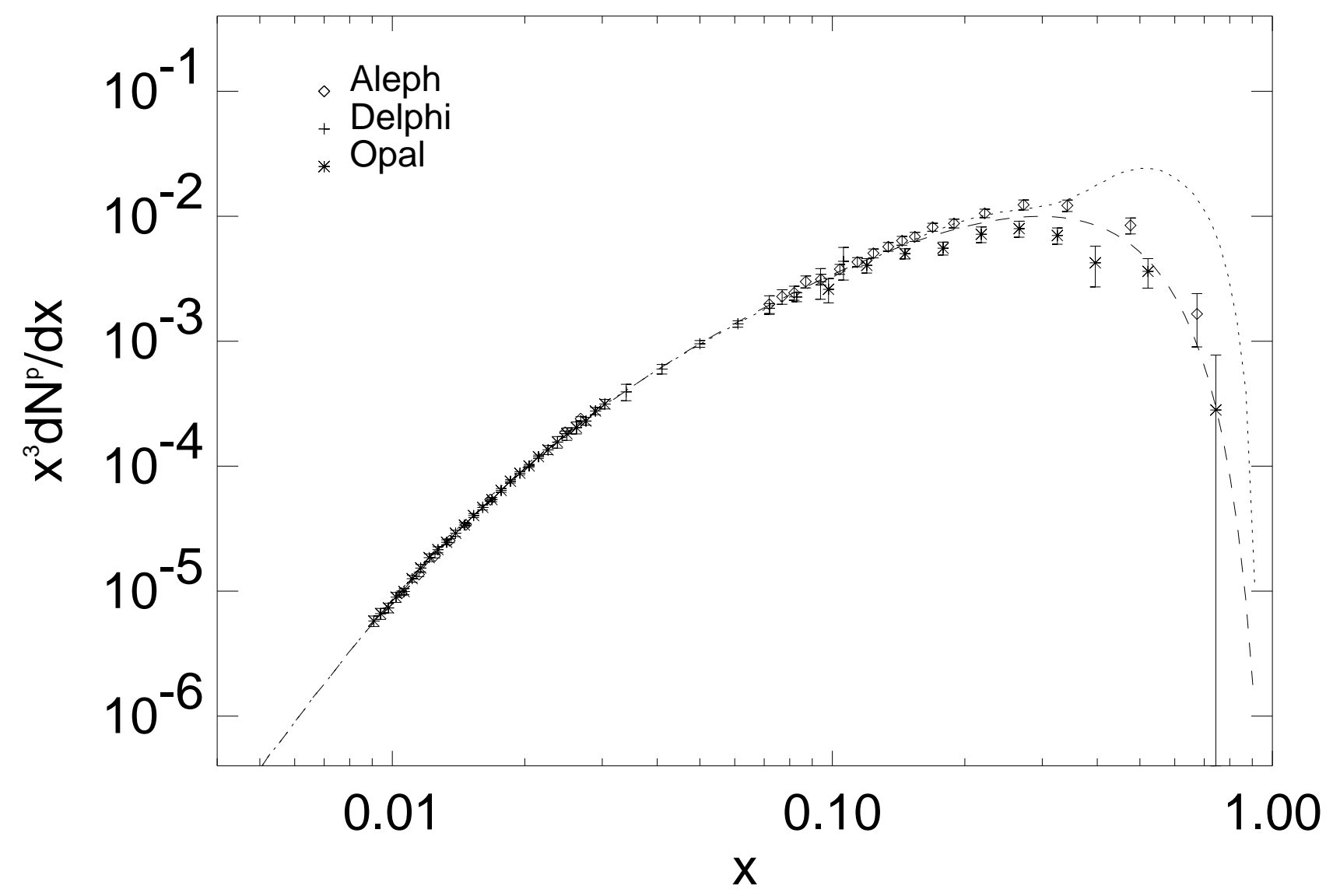

FIG. 1. The fragmentation spectrum of protons at the $Z^{0}$ mass peak, as measured by ALEPH, DELPHI and OPAL at LEP; the dashed line is a parametric fit 86]. The dotted line is the spectrum simulated with HERWIG, illustrating its tendency to overproduce baryons at high $x$. 


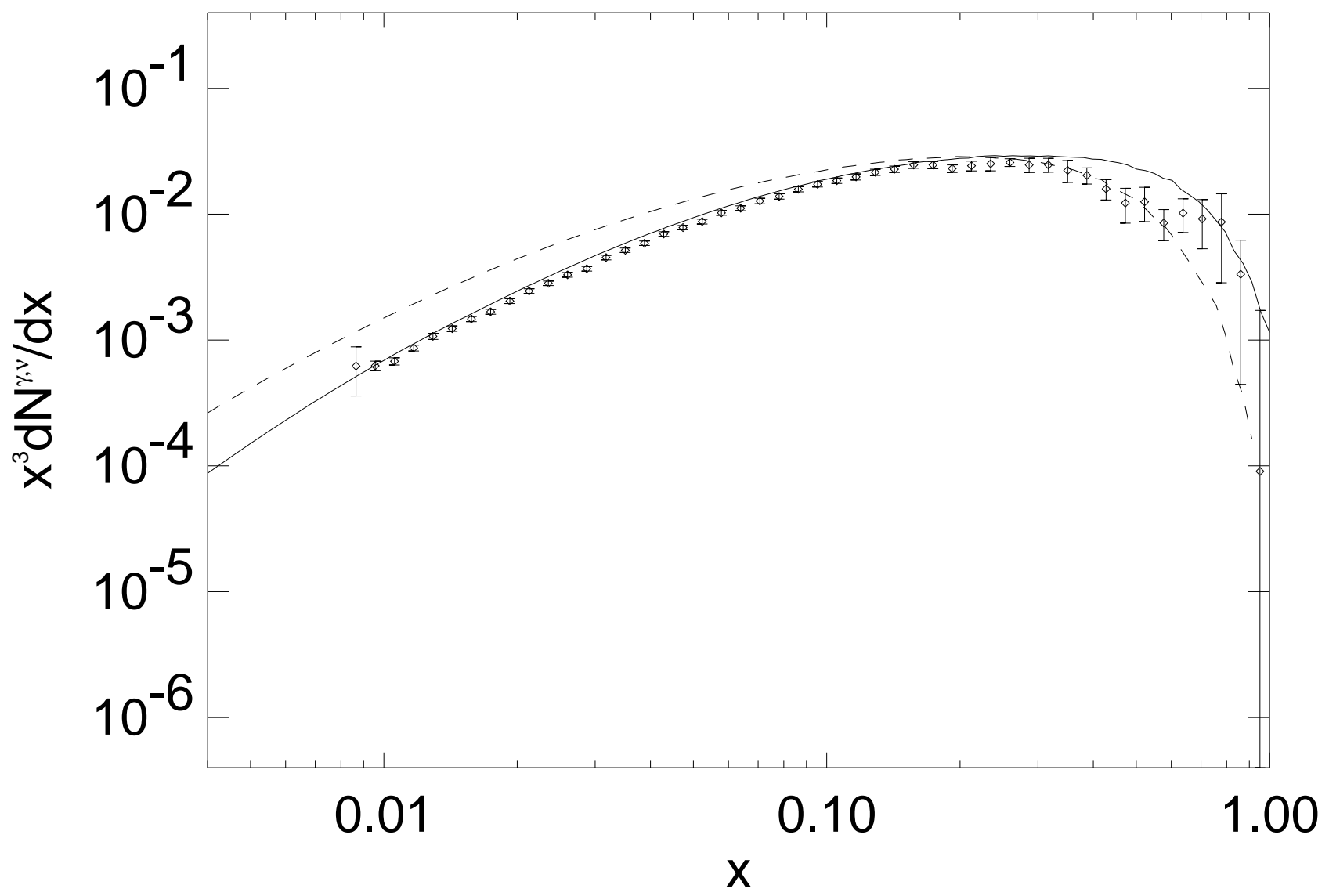

FIG. 2. The fragmentation spectrum of photons at the $Z^{0}$ mass peak, as measured by ALEPH at LEP; the solid line is the spectrum simulated with HERWIG. The fragmentation spectrum of neutrinos from HERWIG is also shown as the dashed line. 


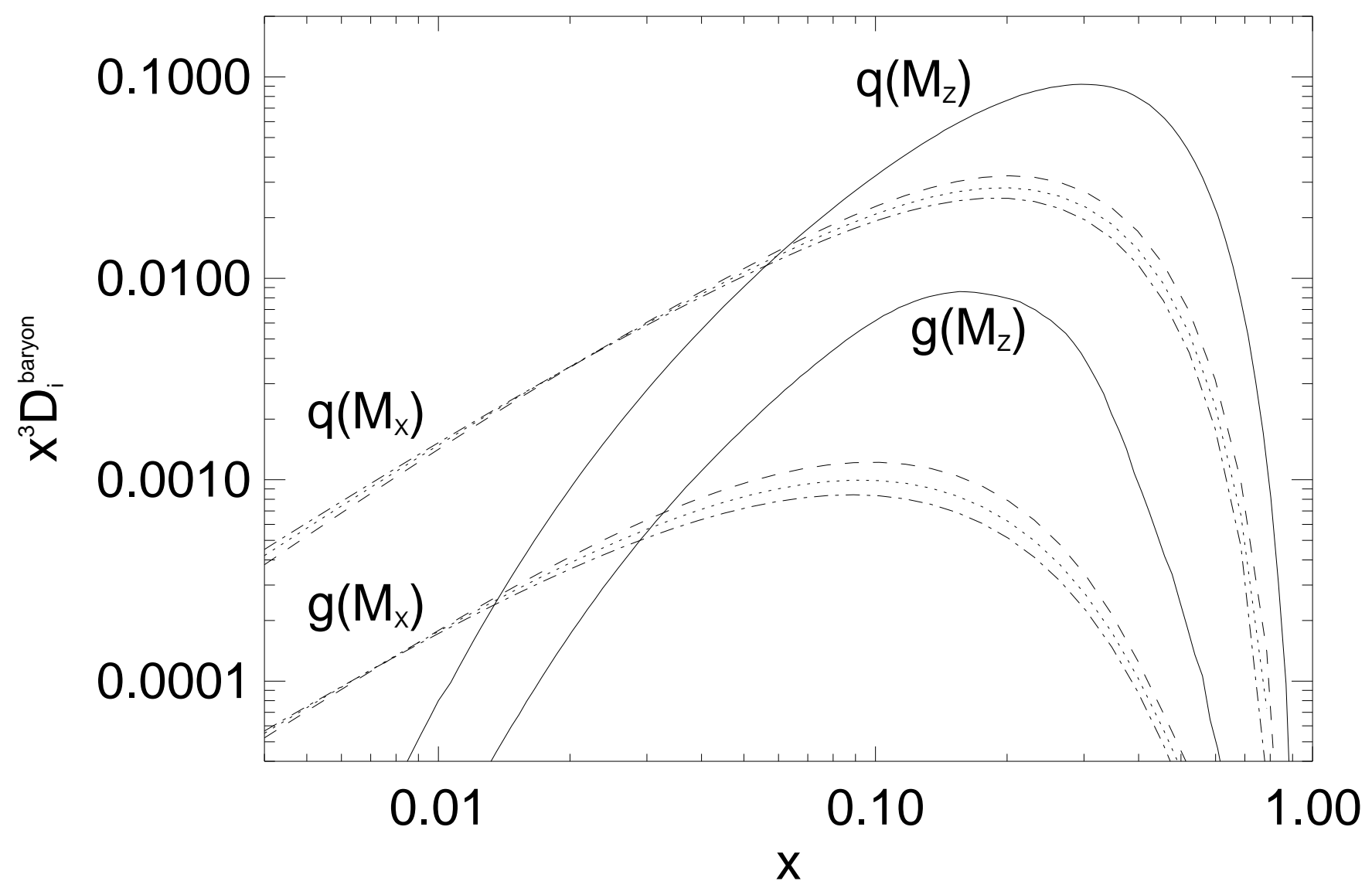

FIG. 3. Standard Model fragmentation functions for baryons from quarks and gluons, at the initial scale $M_{Z}$ (solid lines) and evolved to a decaying particle mass scale of $10^{10} \mathrm{GeV}$ (dashed line), $10^{12} \mathrm{GeV}$ (dotted line) and $10^{14} \mathrm{GeV}$ (dot-dashed line), illustrating scaling violations 


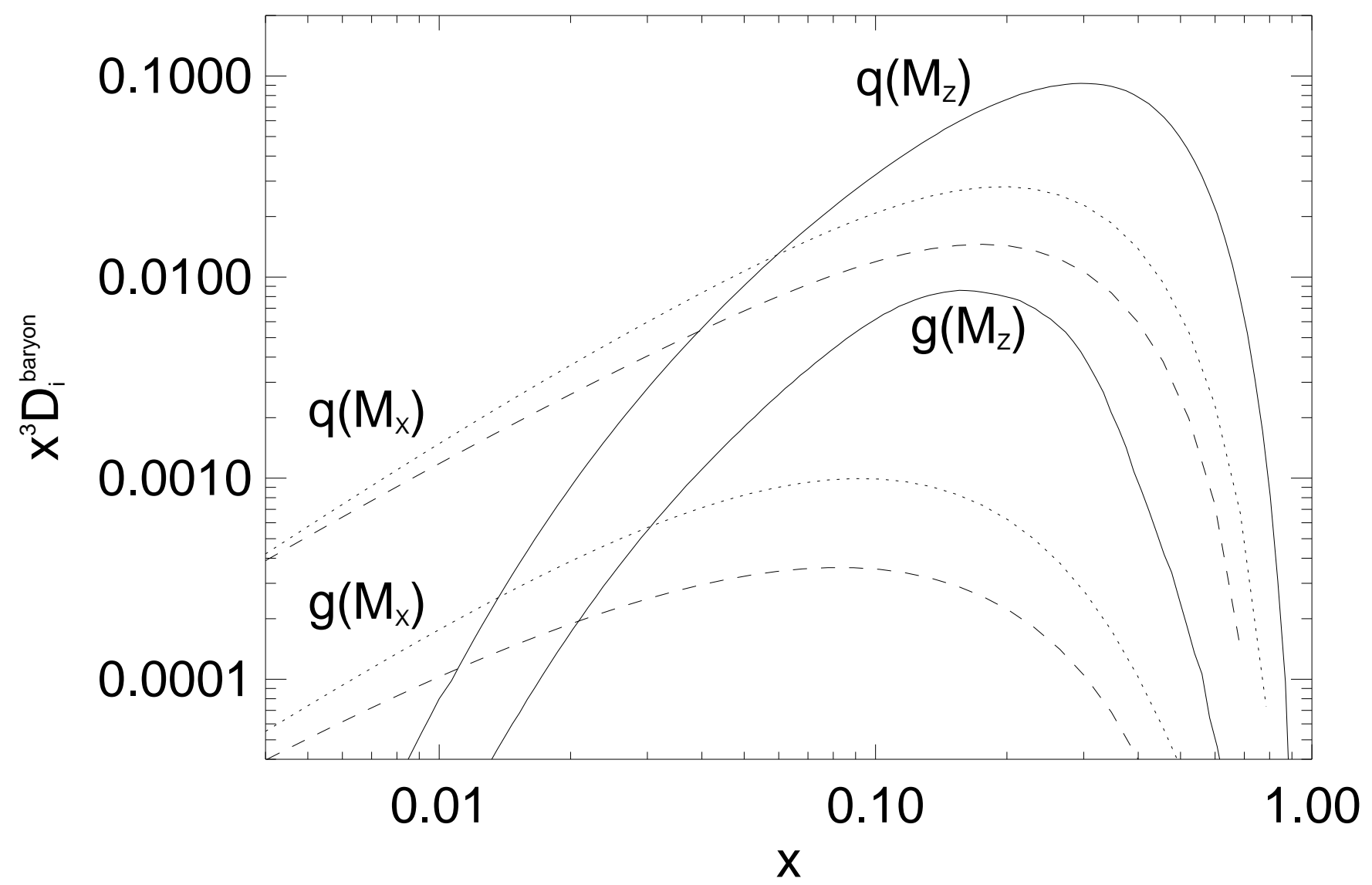

FIG. 4. Fragmentation functions for baryons from quarks and gluons, at the initial scale $M_{Z}$ (solid lines) and evolved to a decaying particle mass scale of $10^{12} \mathrm{GeV}$, for SM evolution (dotted lines), and, the more pronounced, SUSY evolution (dashed lines) taking $M_{\mathrm{SUSY}}=400 \mathrm{GeV}$. 


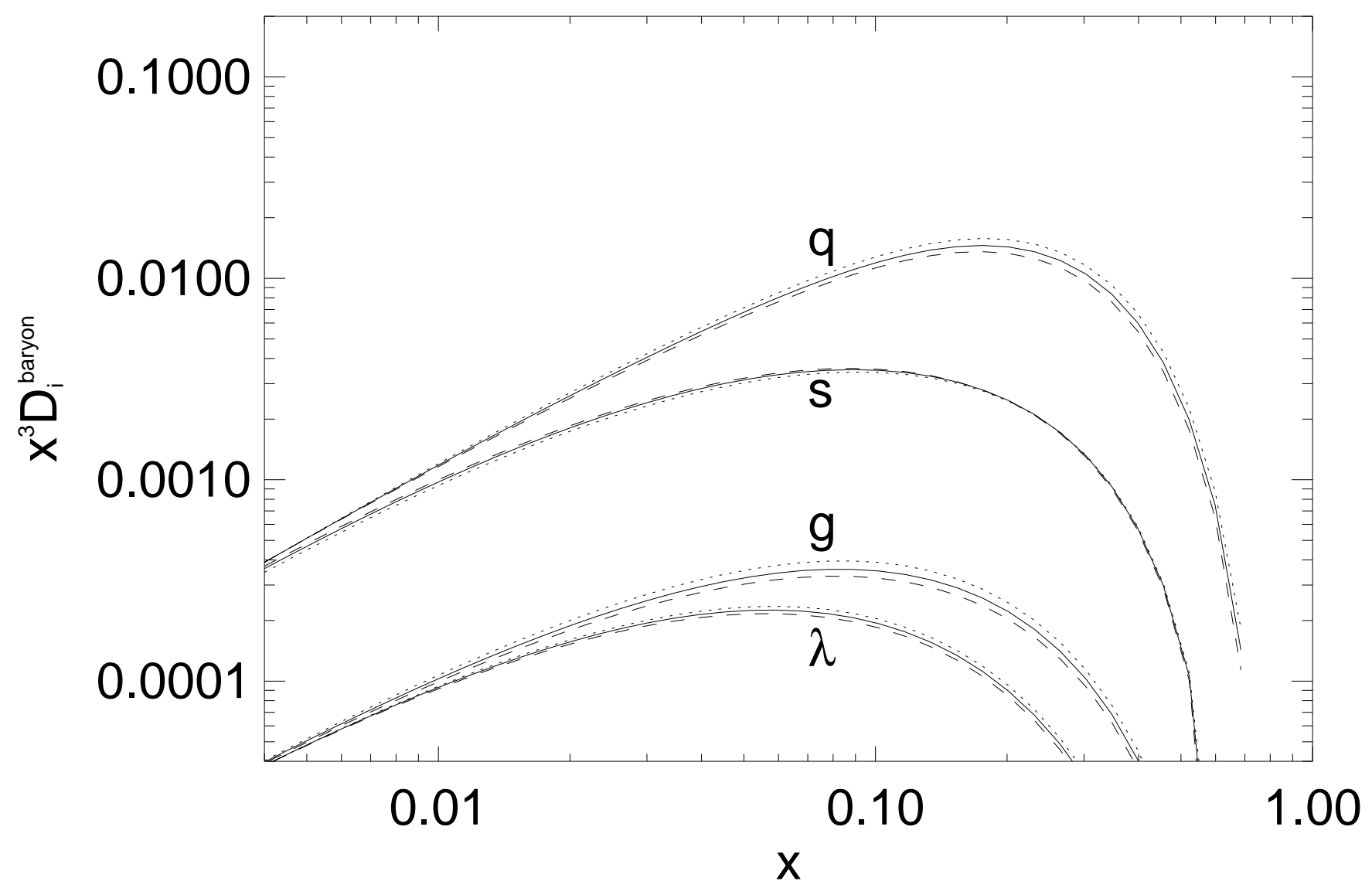

FIG. 5. Dependence of (s)parton fragmentation functions evolved from $M_{Z}$ up to $M_{X}=10^{12} \mathrm{GeV}$ on $M_{\mathrm{SUSY}}=200 \mathrm{GeV}$ (dashed lines), $400 \mathrm{GeV}$ (solid lines), $1 \mathrm{TeV}$ (dotted lines). 

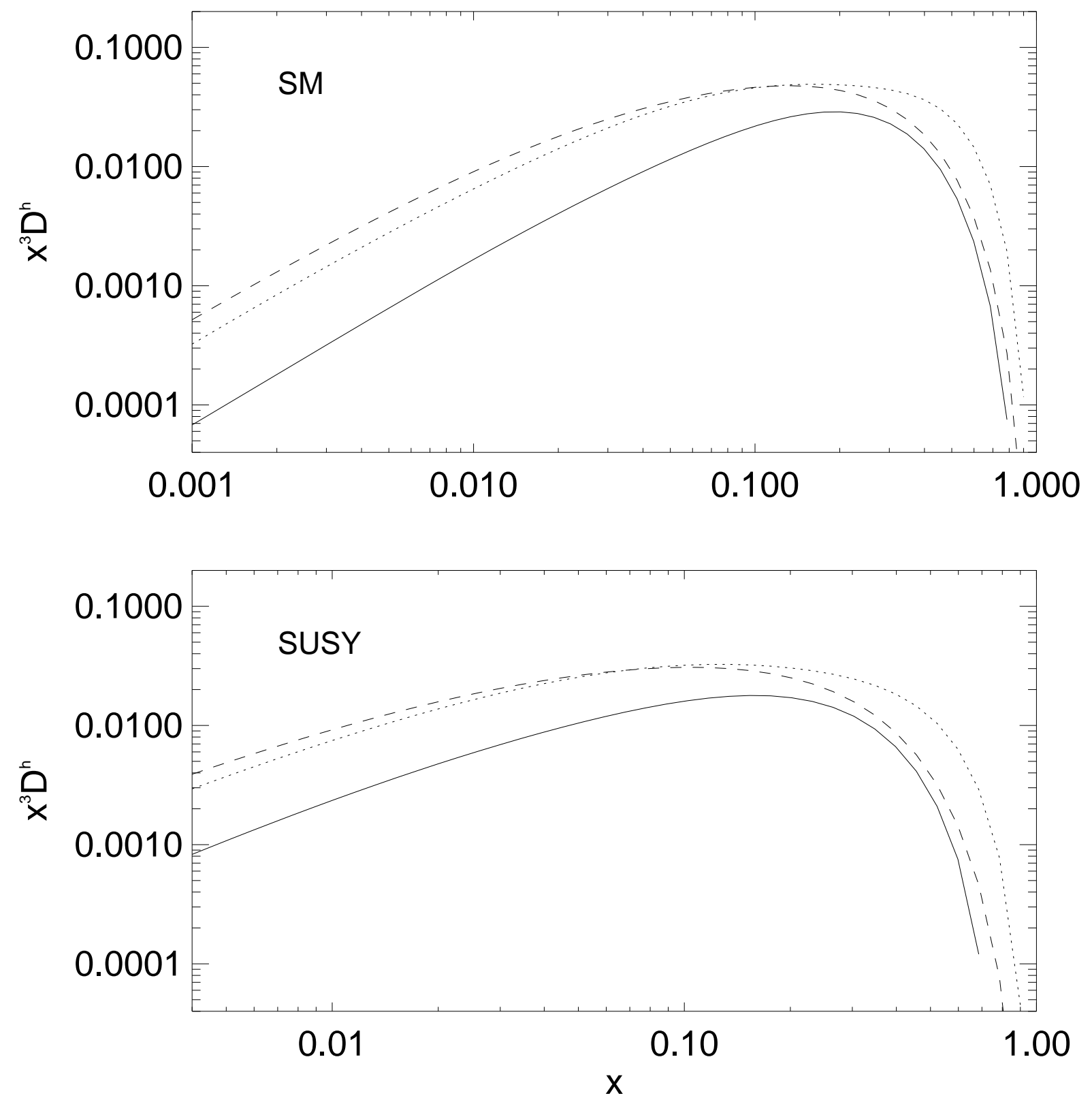

FIG. 6. Fragmentation functions for baryons (solid lines), photons (dotted lines) and neutrinos (dashed lines) evolved from $M_{Z}$ up to $M_{X}=10^{12} \mathrm{GeV}$ for the SM (top panel) and for SUSY with $M_{\mathrm{SUSY}}=400 \mathrm{GeV}$ (bottom panel). 


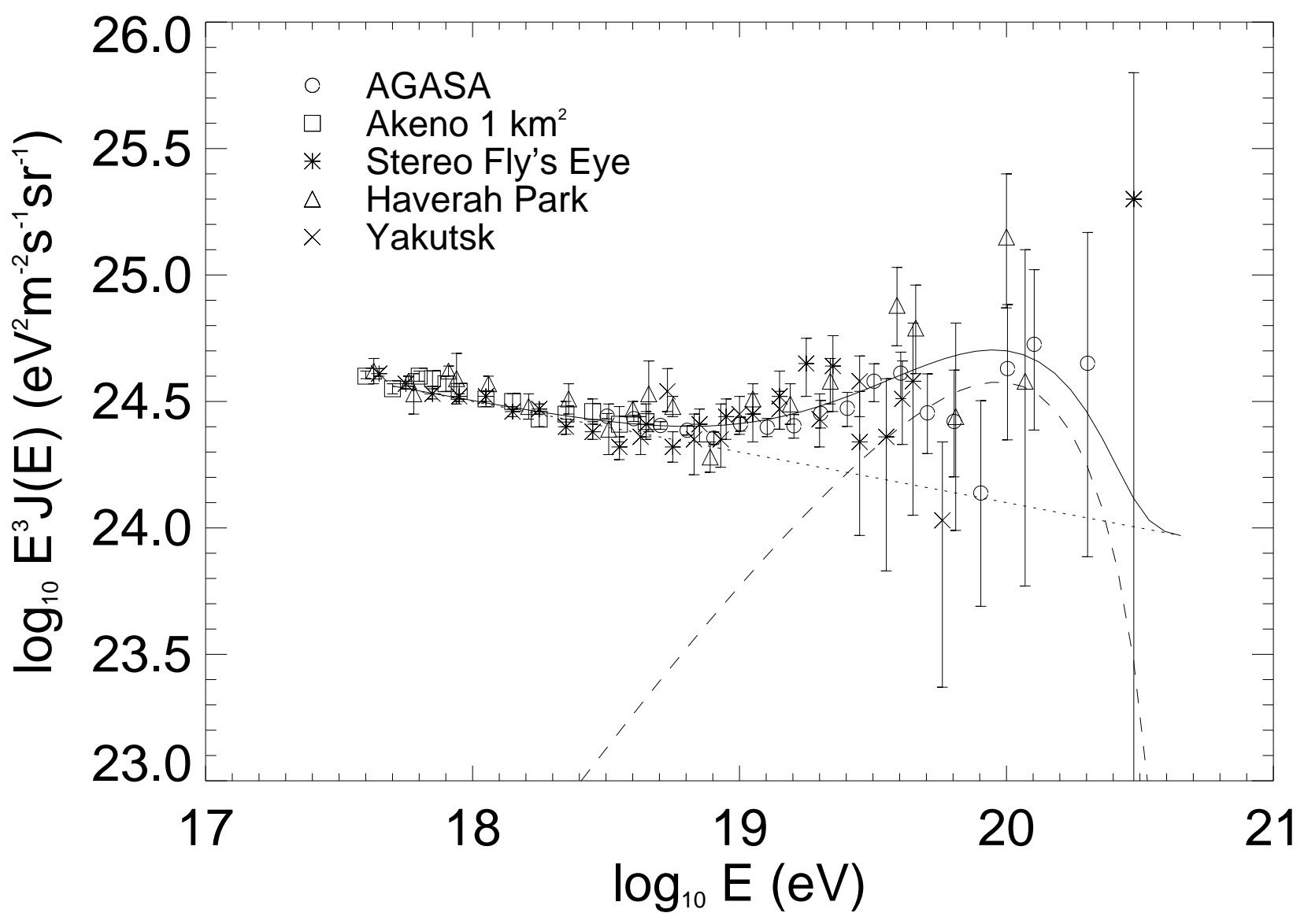

FIG. 7. The best SM evolution fit to the cosmic ray data with a decaying particle mass of $10^{12} \mathrm{GeV}$. The dotted line indicates the extrapolation of the power-law component from lower energies, while the dashed line shows the decay spectrum; the solid line is their sum. 


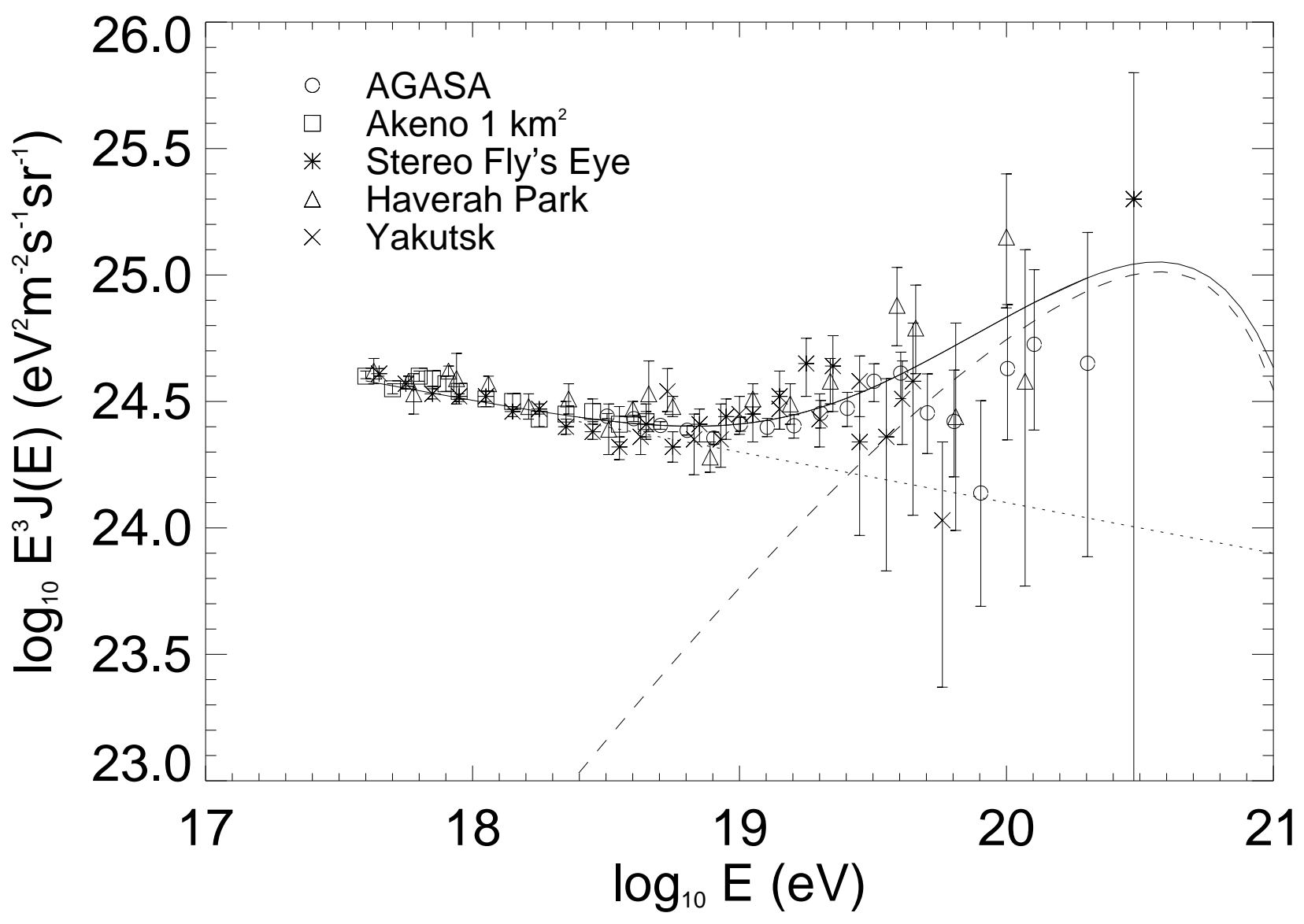

FIG. 8. The best SUSY evolution fit to the cosmic ray data with a decaying particle mass of $5 \times 10^{12} \mathrm{GeV}$. The dotted line indicates the extrapolation of the power-law component from lower energies, while the dashed line shows the decay spectrum; the solid line is their sum. 


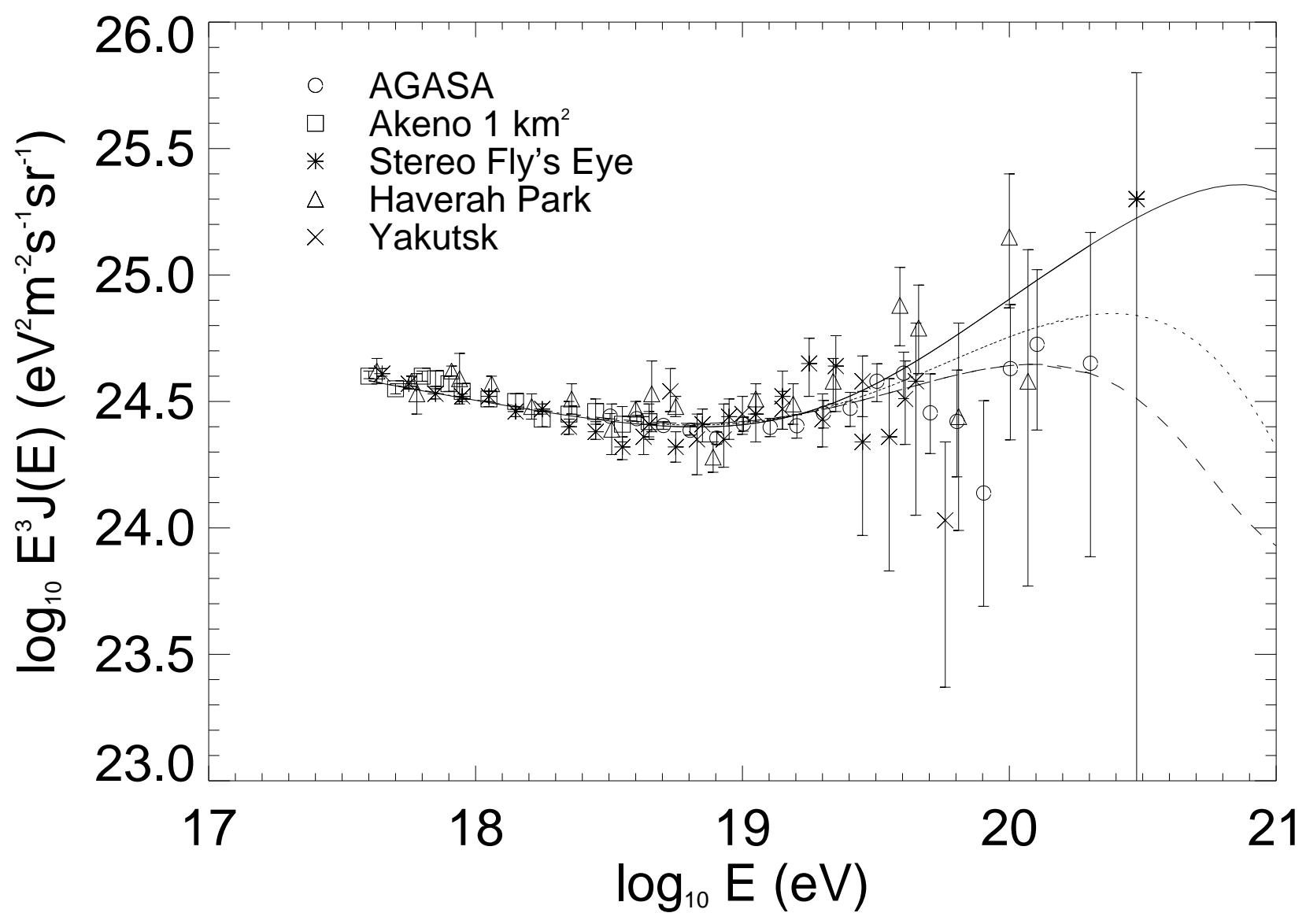

FIG. 9. Cosmic ray data compared with SUSY evolved spectra for a decaying particle mass of $10^{13} \mathrm{GeV}$ with $M_{\mathrm{SUSY}}=400 \mathrm{GeV}$, and many-body decays to $n$ partons: $n=2$ (solid line), $n=8$ (dotted line) and $n=16$ (dashed line). 

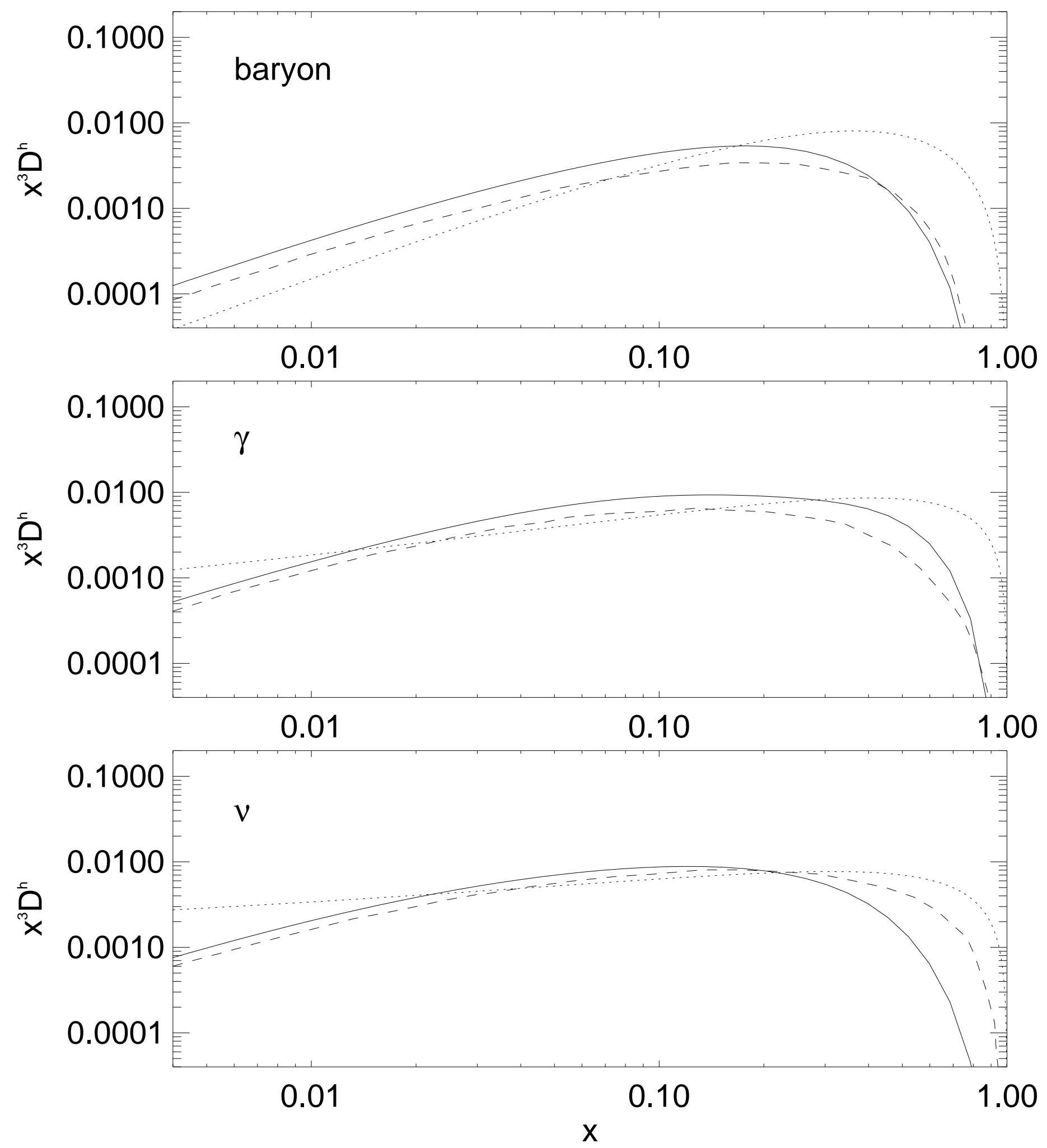

FIG. 10. Comparison of fragmentation functions of baryons (top panel), photons (middle panel) and neutrinos (bottom panel) obtained from (SM) DGLAP evolution in the present work (solid lines) and in Ref. 86] (dashed lines), and using HERWIG [49] (dotted lines). 

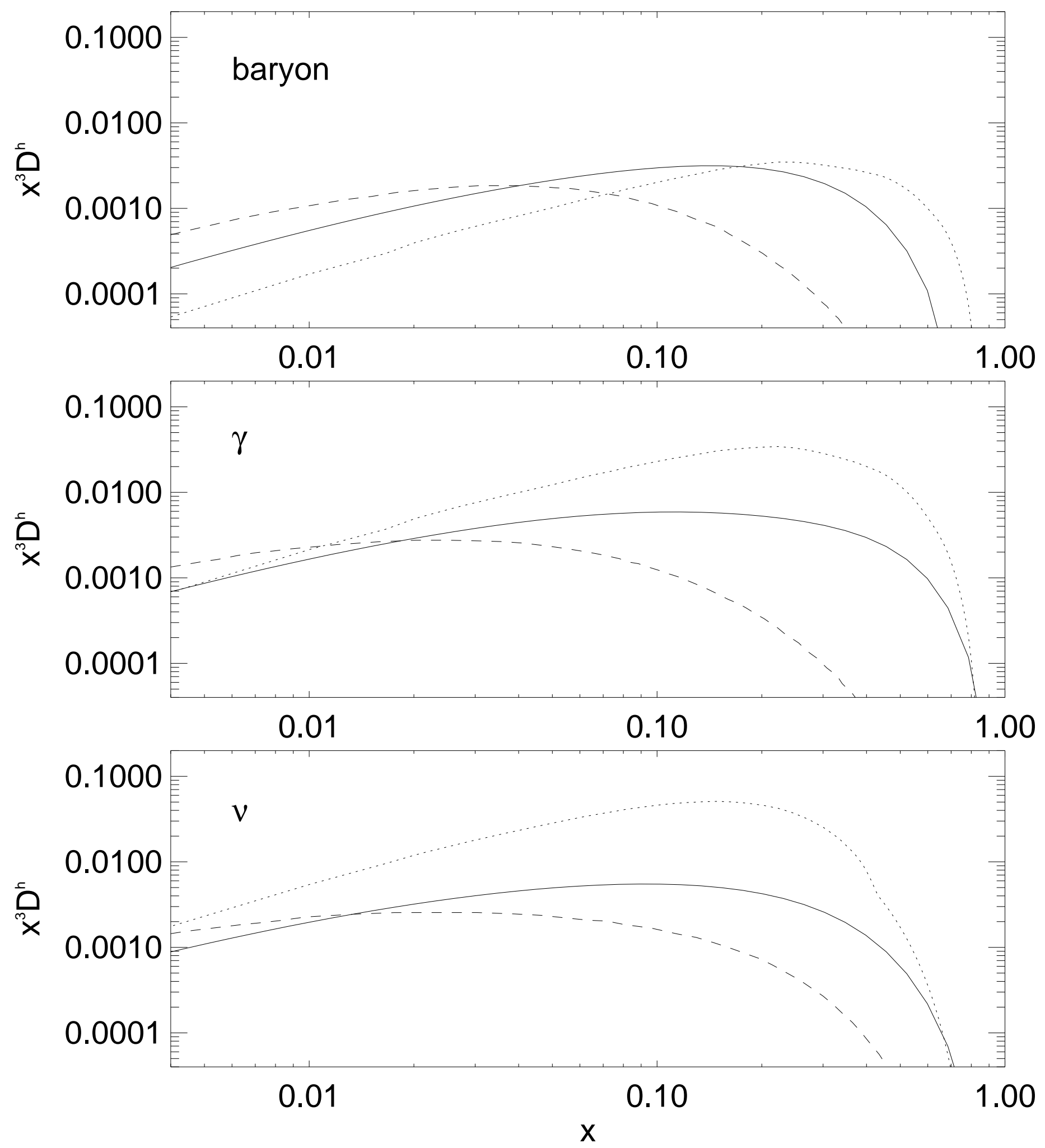

FIG. 11. Comparison of fragmentation functions of baryons (top panel), photons (middle panel) and neutrinos (bottom panel) obtained from (SUSY) DGLAP evolution in the present work (solid lines) and in Ref. 86] (dashed lines), and using a new fragmentation code 96] (dotted lines). 


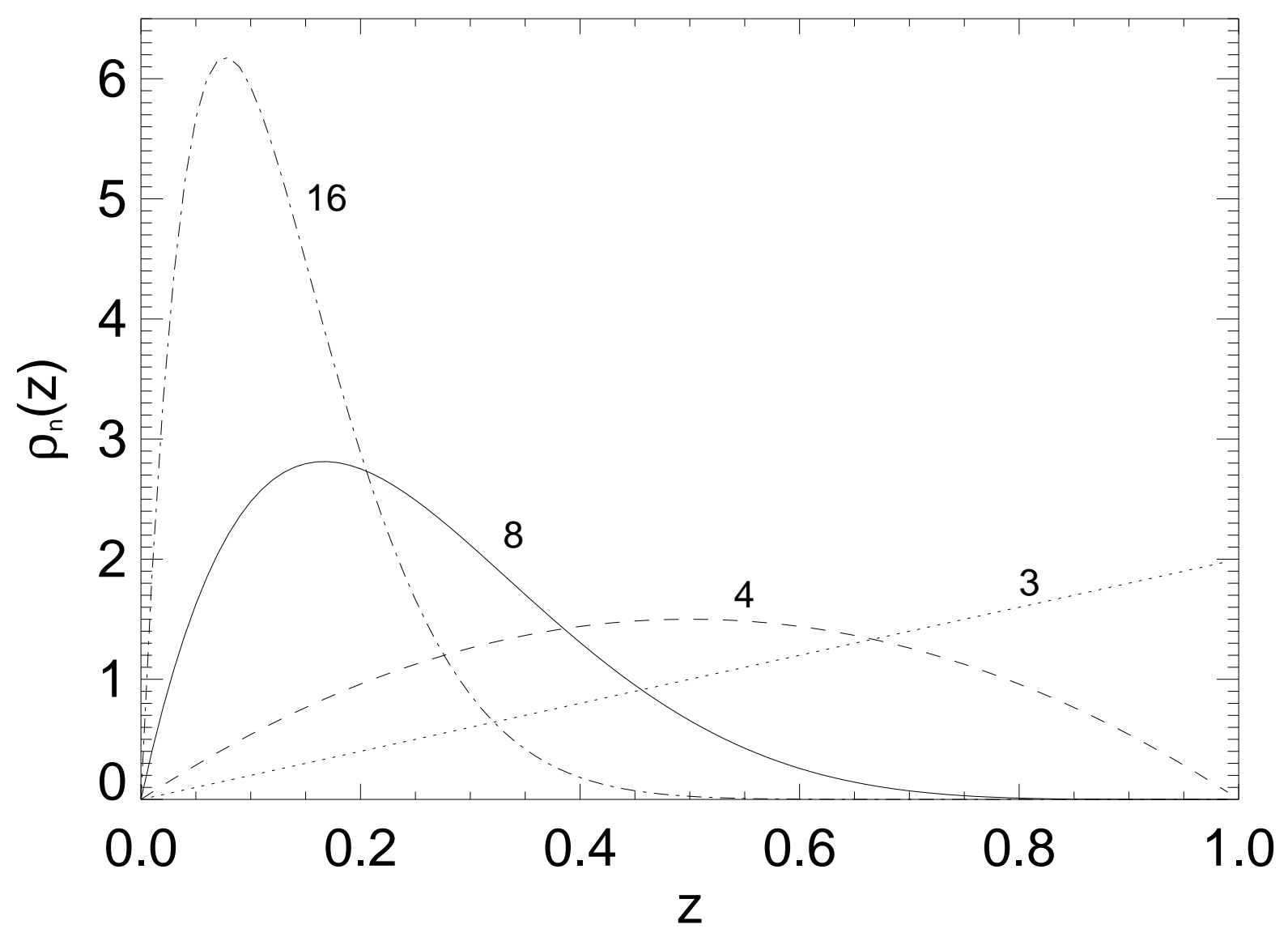

FIG. 12. Final state phase space probability density $\rho_{n}(z)$ for $n=3,4,8,16$. 